\title{
Rotating and orbiting strings in Dp-brane background
}

\author{
Sagar Biswas and Kamal L. Panigrahi \\ Department of Physics, Indian Institute of Technology Kharagpur, \\ 721302, Kharagpur, India \\ E-mail: sbiswas@phy.iitkgp.ernet.in, panigrahi@phy.iitkgp.ernet.in
}

ABSTRACT: We probe the open fundamental strings in Dp-brane $(p=1,3,5)$ backgrounds and find new classes of rotating and orbiting string solutions. We show that for various worldsheet embedding ansatz we get solutions of the string equations of motion that correspond to the well known giant magnon and single spikes, in addition to few new solutions corresponding to the orbiting strings. We make a systematic study of both rigidly rotating and orbiting strings in D1, D3 and D5-brane backgrounds.

KEYWORDS: D-branes, Bosonic Strings

ArXiv EPRINT: 1412.6941 


\section{Contents}

1 Introduction and summary 1

2 Probing the fundamental string in Dp-brane backgrounds 3

3 Rotating and orbiting strings in D3-brane background $\quad 8$

$\begin{array}{ll}3.1 \text { Rotating string solutions: constant }\left(r, \phi_{3}\right) & 9\end{array}$



$\begin{array}{lll}3.1 .2 & \text { Single spike case } & 11\end{array}$

$\begin{array}{ll}3.2 & \text { Rotating string solution: constant }\left(r, \phi_{1}\right) \\ 3.3 & \text { Orbiting string solution: constant }\left(\phi_{1}, \phi_{3}\right)\end{array}$

$\begin{array}{lll}3.3 & \text { Orbiting string solution: constant }\left(\phi_{1}, \phi_{3}\right) & 13\end{array}$

4 Strings in D5-brane background $\quad \mathbf{1 5}$

$\begin{array}{ll}4.1 \text { Rotating string solution: constant }(r) & 16\end{array}$

$\begin{array}{lll}4.2 & \text { Orbiting string solution: constant }\left(\phi_{1}\right) & 18\end{array}$

5 Strings in D1-brane background $\quad 19$

5.1 Rotating string solution: constant $\left(r, \phi_{3}, \phi_{5}\right) \quad 21$

5.2 Orbiting string solution: constant $\left(\phi_{1}, \phi_{3}, \phi_{5}\right) \quad 22$

6 Conclusion $\quad 23$

\section{Introduction and summary}

The celebrated AdS/CFT duality relates weakly coupled type IIB string theory on the $\mathrm{AdS}_{5} \times S^{5}$ to strongly coupled large $N \mathcal{N}=4$ super Yang-Mills (SYM) theory in $3+1$ dimensional spacetime [1], [2], [3]. Proving the conjecture beyond supergravity approximations is quite a hard problem because of the unavailability of fully quantized string spectrum in the AdS side. However over the past few years there has been lot of attempts in understanding the duality in various sub sectors of the theories. It has been noticed that in large angular momentum region one can use the semiclassical approximation to find the string spectrum and eventually look for the string state-operator matching more precisely [4], [5]. With the further realization that the counting of gauge invariant operators from gauge theory side can be elegantly formulated in terms of an integrable spin chain [6], [7], [8], [9], [10], it has been established that integrability played an important role on both sides of the duality, since the dual string theory is integrable in the semiclassical limit. In this context, in [18], a special limit was put forth using which both sides of the duality was analyzed in detail. In particular, the spectrum on the field theory side was shown to be consists of an elementary excitation, the so called magnon which carries a momentum $p$ along the finitely or infinitely long spin chain. The dual string state, derived from the 
rigidly rotating string in the $\mathbb{R} \times S^{3}$ presenting the same dispersion relation in the large 't Hooft limit, is known as the giant magnon. A more general class of rigidly rotating string solutions were also proposed in [16] which are dual to a higher twist operators in the boundary field theory. These kind of solutions are called spiky strings. In addition to the rigidly rotating strings, the spinning and pulsating strings have also been found out to have exact correspondence with some dual operators in the gauge theory [14], [15]. Since then a fairly large class of rotating and pulsating string solutions in various asymptotically AdS and non-AdS backgrounds have been studied and the dual operators have also been examined carefully over the past few years [11]-[38].

In the recent past, the integrability of the classical string motion in curved $\mathrm{D} p$-brane background has been explored in [36] in an attempt to understand the integrability of the full string equations of motion. It was shown that though the point like string equations are integrable, the equations describing an extended string in the complete D-brane background are not. We wish to further shed light on this, by probing the fundamental string in various Dp-branes of type IIB string theory and look for various rigidly rotating and orbiting string solutions. It is well known that among various Dp branes only D3 brane is integrable in the near horizon limit as it produces $\mathrm{AdS}_{5} \times S^{5}$ geometry. We have found the known giant magnons and spikes like solutions for the string in semiclassical limit, whose dual operators are well identified. Along with these, we find out other kind of rotating string solutions in the D3-brane background. Infact both kind of solutions are obtained by looking at consistent worldsheet embedding. Moreover, we find out new orbiting solutions for the string in D3-brane background.

We also try to find some rigidly rotating and orbiting string solutions in D5 and D1 brane background. The string solutions in these backgrounds are non integrable in its full generalization as studied in [36], but we manage to extract some nice results by considering different subspaces of the full space. Such an attempt was done in [38] in the context of the so called supergravity puff field theory background. The reason why one should expect to get some viable solution by considering a particular subspace can be understood as follows. We choose our ansatz in such a way that all non isometry coordinates becomes variables. For general background, by solving the string equation of motions, one get highly coupled and nonlinear differential equations for these variables which are hard to solve. But, by looking at the Virasoro one can get an idea that some kind of solution is possible if we keep only one variable and put the rest as constants. We can choose different types of subspaces. As the variables corresponds to non-isometric coordinates, putting them as constants will impose severe non trivial constraints on the system, which can be easily obtained from the corresponding equation of motion. In other words putting these variables as constant are the solutions of the equation of motion provided that they satisfy the constraint equations. So, the solutions we present for a particular subspace are valid in some particular constraint space. The rest of the paper has been organised as follows. In section 2 we write the most general string equation of motion and Virasoro constraints in near horizon Dp-brane backrgound. Section 3 is devoted to the study of F-string in the D3-brane background. In addition to finding the usual giant magnon and single spike solutions we find few general rotating strings with several angular momenta 
and further the orbiting solutions. We comment on the possible field theory operators they might correspond to. In section 4 we study the solutions to the string equations of motion in the D5-brane background and find a large class of solutions. In section 5 we study the rigidly rotating and orbiting string solutions in D1-brane background. Finally in section 6 we present our conclusions with some remarks.

\section{Probing the fundamental string in Dp-brane backgrounds}

The low energy background field solutions describing a stack of $k$ Dp-branes of tendimensional supergravity is given by

$$
\begin{aligned}
d s^{2} & =h^{-\frac{1}{2}}(r)\left[-d t^{2}+\sum_{i=1}^{p} d x_{i}^{2}\right]+h^{\frac{1}{2}}(r) \sum_{i=p+1}^{9} d y_{i}^{2}, \\
e^{\phi} & =h^{\frac{3-p}{4}}, \quad C_{0 \cdots p}=1-\frac{1}{h(r)},
\end{aligned}
$$

where $C_{p+1}$ is the $(p+1)$ - dimensional Ramond-Ramond (RR) form which couples to Dpbrane, and $\phi$ is the dilaton. When $p<7$ the harmonic function $h(r)$ is explicitly given by $h(r)=1+\frac{k}{r^{7-p}}$ with $r^{2}=y_{p+1}^{2}+\cdots+d y_{9}^{2}$ and $k=c_{p} g_{s} N l_{s}^{7-p}$ being $c_{p}=(2 \sqrt{\pi})^{5-p} \Gamma\left(\frac{7-p}{2}\right)$. The transverse space part of the metric (2.1) can be written as,

$$
\begin{aligned}
\sum_{i=p+1}^{9} d y_{i}^{2} & =d r^{2}+r^{2} d \Omega_{8-p}^{2}=d r^{2}+r^{2}\left(d \phi_{1}^{2}+\sin ^{2} \phi_{1} d \phi_{2}^{2}+\cos ^{2} \phi_{1} d \Omega_{6-p}^{2}\right) \\
& =d r^{2}+r^{2}\left[d \phi_{1}^{2}+\sin ^{2} \phi_{1} d \phi_{2}^{2}+\cos ^{2} \phi_{1}\left(d \phi_{3}^{2}+\sin ^{2} \phi_{3} d \phi_{4}^{2}+\cos ^{2} \phi_{3} d \Omega_{4-p}^{2}\right)\right]
\end{aligned}
$$

We will be interested in Dp-brane $(p=1,3,5)$ backgrounds, so one should stop when the suffix of $\Omega$ becomes $2-p$. Now we can rewrite the metric for Dp-branes, $(p=1,3,5)$,

$$
\begin{aligned}
d s^{2}= & h^{-\frac{1}{2}}(r)\left[-d t^{2}+\sum_{i=1}^{p} d x_{i}^{2}\right]+h^{\frac{1}{2}}(r)\left[d r^{2}+r^{2}\left\{d \phi_{2-p}^{2}+\sin ^{2} \phi_{2-p} d \phi_{3-p}^{2}\right.\right. \\
& +\cos ^{2} \phi_{2-p}\left(d \phi_{4-p}^{2}+\sin ^{2} \phi_{4-p} d \phi_{5-p}^{2}+\cos ^{2} \phi_{4-p}\left(d \phi_{6-p}^{2}\right.\right. \\
& \left.\left.\left.\left.+\sin ^{2} \phi_{6-p} d \phi_{7-p}^{2}+\cos ^{2} \phi_{6-p} d \phi_{8-p}^{2}\right)\right)\right\}\right], \\
e^{\phi}= & h^{\frac{3-p}{4}}, \quad C_{0 \cdots p}=1-\frac{1}{h(r)} .
\end{aligned}
$$

In near horizon limit $r \rightarrow 0$, the metric, dilaton and $\mathrm{RR} p$-form field become,

$$
\begin{aligned}
d s^{2}= & \frac{r^{\frac{7-p}{2}}}{\sqrt{k}}\left[-d t^{2}+\sum_{i=1}^{p} d x_{i}^{2}\right]+\frac{\sqrt{k}}{r^{\frac{7-p}{2}}}\left[d r^{2}+r^{2}\left\{d \phi_{2-p}^{2}+\sin ^{2} \phi_{2-p} d \phi_{3-p}^{2}\right.\right. \\
& +\cos ^{2} \phi_{2-p}\left(d \phi_{4-p}^{2}+\sin ^{2} \phi_{4-p} d \phi_{5-p}^{2}+\cos ^{2} \phi_{4-p}\left(d \phi_{6-p}^{2}\right.\right. \\
& \left.\left.\left.\left.+\sin ^{2} \phi_{6-p} d \phi_{7-p}^{2}+\cos ^{2} \phi_{6-p} d \phi_{8-p}^{2}\right)\right)\right\}\right] \\
e^{\phi}= & \left(\frac{k}{r^{7-p}}\right)^{\frac{3-p}{4}}, \quad C_{0 \cdots p}=1-\frac{r^{7-p}}{k} .
\end{aligned}
$$


Now rescaling $t \rightarrow \sqrt{k} t$ and $x_{i} \rightarrow \sqrt{k} x_{i}$, the metric in equation (2.4) becomes,

$$
\begin{aligned}
d s^{2}= & \sqrt{k} r^{\frac{7-p}{2}}\left[-d t^{2}+\sum_{i=1}^{p} d x_{i}^{2}\right]+\frac{\sqrt{k}}{r^{\frac{7-p}{2}}}\left[d r^{2}+r^{2}\left\{d \phi_{2-p}^{2}+\sin ^{2} \phi_{2-p} d \phi_{3-p}^{2}\right.\right. \\
& +\cos ^{2} \phi_{2-p}\left(d \phi_{4-p}^{2}+\sin ^{2} \phi_{4-p} d \phi_{5-p}^{2}+\cos ^{2} \phi_{4-p}\left(d \phi_{6-p}^{2}\right.\right. \\
& \left.\left.\left.\left.+\sin ^{2} \phi_{6-p} d \phi_{7-p}^{2}+\cos ^{2} \phi_{6-p} d \phi_{8-p}^{2}\right)\right)\right\}\right] .
\end{aligned}
$$

The Polyakov action of the F-string in the background (2.5) is given by,

$$
S=-\frac{1}{4 \pi \alpha^{\prime}} \int d \sigma d \tau\left[\sqrt{-\gamma} \gamma^{\alpha \beta} g_{M N} \partial_{\alpha} X^{M} \partial_{\beta} X^{N}\right]
$$

where $\gamma^{\alpha \beta}$ is the world-sheet metric . In conformal gauge (i.e. $\sqrt{-\gamma} \gamma^{\alpha \beta}=\eta^{\alpha \beta}$ ) with $\eta^{\tau \tau}=-1, \eta^{\sigma \sigma}=1$ and $\eta^{\tau \sigma}=\eta^{\sigma \tau}=0$, the Polyakov action in the above background takes the form,

$$
\begin{aligned}
S= & -\frac{\sqrt{k}}{4 \pi} \int d \sigma d \tau\left[r^{\frac{7-p}{2}}\left\{-\left({t^{\prime}}^{2}-\dot{t}^{2}\right)+{x_{i}^{\prime}}^{2}-\dot{x}_{i}^{2}\right\}+\frac{1}{r^{\frac{7-p}{2}}}\left({r^{\prime}}^{2}-\dot{r}^{2}\right)\right. \\
& +r^{\frac{p-3}{2}}\left\{\left(\phi_{2-p}^{\prime}{ }^{2}-\dot{\phi}_{2-p}^{2}\right)+\sin ^{2} \phi_{2-p}\left(\phi_{3-p}^{\prime}{ }^{2}-\dot{\phi}_{3-p}^{2}\right)+\cos ^{2} \phi_{2-p}\left(\phi_{4-p}^{\prime}{ }^{2}-\dot{\phi}_{4-p}^{2}\right)\right. \\
& +\cos ^{2} \phi_{2-p} \sin ^{2} \phi_{4-p}\left(\phi_{5-p}^{\prime}{ }^{2}-\dot{\phi}_{5-p}^{2}\right)+\cos ^{2} \phi_{2-p} \cos ^{2} \phi_{4-p}\left(\phi_{6-p}^{\prime}{ }^{2}-\dot{\phi}_{6-p}^{2}\right) \\
& +\cos ^{2} \phi_{2-p} \cos ^{2} \phi_{4-p} \sin ^{2} \phi_{6-p}\left(\phi_{7-p}^{\prime}{ }^{2}-\dot{\phi}_{7-p}^{2}\right) \\
& \left.\left.+\cos ^{2} \phi_{2-p} \cos ^{2} \phi_{4-p} \cos ^{2} \phi_{6-p}\left(\phi_{8-p}^{\prime}{ }^{2}-\dot{\phi}_{8-p}^{2}\right)\right\}\right]
\end{aligned}
$$

where 'dots' and 'primes' denote the derivative with respect to $\tau$ and $\sigma$ respectively. Now we choose our ansatz in such a way that the non isometry coordinates become variables,

$$
\begin{array}{rlrlrl}
t & =\tau+h_{0}(y), & x_{i} & =\nu_{i}\left(\tau+h_{i}(y)\right), & r=r(y), \\
\phi_{2-p} & =\phi_{2-p}(y), & \phi_{3-p} & =\omega_{3-p}\left(\tau+g_{3-p}(y)\right), \quad \phi_{4-p}=\phi_{4-p}(y), \\
\phi_{5-p} & =\omega_{5-p}\left(\tau+g_{5-p}(y)\right), & \phi_{6-p} & =\phi_{6-p}(y), \\
\phi_{7-p} & =\omega_{7-p}\left(\tau+g_{7-p}(y)\right), & \phi_{8-p} & =\omega_{8-p}\left(\tau+g_{8-p}(y)\right), &
\end{array}
$$

where $y=\sigma-v \tau$ and $i=1, \cdots, p$. Variation of the action with respect to $X^{M}$ gives us the following equations of motion

$$
2 \partial_{\alpha}\left(\eta^{\alpha \beta} \partial_{\beta} X^{N} g_{K N}\right)-\eta^{\alpha \beta} \partial_{\alpha} X^{M} \partial_{\beta} X^{N} \partial_{K} g_{M N}=0,
$$

and variation with respect to the metric gives the two Virasoro constraints,

$$
\begin{aligned}
g_{M N}\left(\partial_{\tau} X^{M} \partial_{\tau} X^{N}+\partial_{\sigma} X^{M} \partial_{\sigma} X^{N}\right) & =0, \\
g_{M N}\left(\partial_{\tau} X^{M} \partial_{\sigma} X^{N}\right) & =0 .
\end{aligned}
$$


Next we have to solve these equations by the ansatz we have proposed above in (2.8). Solving for the isometry coordinates $t, x_{i}, \phi_{3-p}, \phi_{5-p}, \phi_{7-p}$ and $\phi_{8-p}$ respectively, we get,

$$
\begin{aligned}
\frac{\partial h_{0}}{\partial y} & =\frac{1}{1-v^{2}}\left[r^{\frac{p-7}{2}} c_{0}-v\right], \\
\frac{\partial h_{i}}{\partial y} & =\frac{1}{1-v^{2}}\left[r^{\frac{p-7}{2}} c_{i}-v\right], \\
\frac{\partial g_{3-p}}{\partial y} & =\frac{1}{1-v^{2}}\left[\frac{d_{3-p}}{r^{\frac{p-3}{2}} \sin ^{2} \phi_{2-p}}-v\right], \\
\frac{\partial g_{5-p}}{\partial y} & =\frac{1}{1-v^{2}}\left[\frac{d_{5-p}}{r^{\frac{p-3}{2}} \cos ^{2} \phi_{2-p} \sin ^{2} \phi_{4-p}}-v\right], \\
\frac{\partial g_{7-p}}{\partial y} & =\frac{1}{1-v^{2}}\left[\frac{d_{7-p}}{r^{\frac{p-3}{2}} \cos ^{2} \phi_{2-p} \cos ^{2} \phi_{4-p} \sin ^{2} \phi_{6-p}}-v\right], \\
\frac{\partial g_{8-p}}{\partial y} & =\frac{1}{1-v^{2}}\left[\frac{d_{8-p}}{r^{\frac{p-3}{2}} \cos ^{2} \phi_{2-p} \cos ^{2} \phi_{4-p} \cos ^{2} \phi_{6-p}}-v\right],
\end{aligned}
$$

where $c_{0}, c_{i}(i=1, \cdots, p), d_{3-p}, d_{5-p}, d_{7-p}$ and $d_{8-p}$ are integration constants. Now we want to solve the equations of motion corresponding to the non isometry coordinates one by one. First for $\phi_{6-p}$ we get,

$$
\begin{aligned}
(1- & \left.v^{2}\right)^{2} \frac{\partial}{\partial y}\left[r^{\frac{p-3}{2}} \cos ^{2} \phi_{2-p} \cos ^{2} \phi_{4-p} \frac{\partial \phi_{6-p}}{\partial y}\right] \\
= & r^{\frac{p-3}{2}} \cos ^{2} \phi_{2-p} \cos ^{2} \phi_{4-p} \sin \phi_{6-p} \cos \phi_{6-p}\left[\frac{\omega_{7-p}^{2} d_{7-p}^{2}}{r^{p-3} \cos ^{4} \phi_{2-p} \cos ^{4} \phi_{4-p} \sin ^{4} \phi_{6-p}}\right. \\
& \left.-\frac{\omega_{8-p}^{2} d_{8-p}^{2}}{r^{p-3} \cos ^{4} \phi_{2-p} \cos ^{4} \phi_{4-p} \cos ^{4} \phi_{6-p}}+\omega_{8-p}^{2}-\omega_{7-p}^{2}\right]
\end{aligned}
$$

For $\phi_{4-p}$ we get,

$$
\begin{gathered}
\left(1-v^{2}\right)^{2} \frac{\partial}{\partial y}\left[r^{\frac{p-3}{2}} \cos ^{2} \phi_{2-p} \frac{\partial \phi_{4-p}}{\partial y}\right]=r^{\frac{p-3}{2}} \cos ^{2} \phi_{2-p} \sin \phi_{4-p} \cos \phi_{4-p} \\
\times\left[\frac{\omega_{5-p}^{2} d_{5-p}^{2}}{r^{p-3} \cos ^{4} \phi_{2-p} \sin _{4-p}^{4}}-\frac{\omega_{7-p}^{2} d_{7-p}^{2}}{r^{p-3} \cos ^{4} \phi_{2-p} \cos ^{4} \phi_{4-p} \sin ^{2} \phi_{6-p}}\right. \\
\quad-\frac{\omega_{8-p}^{2} d_{8-p}^{2}}{r^{p-3} \cos ^{4} \phi_{2-p} \cos ^{4} \phi_{4-p} \cos ^{2} \phi_{6-p}}-\omega_{5-p}^{2}+\omega_{7-p}^{2} \sin ^{2} \phi_{6-p} \\
\left.\quad+\omega_{8-p}^{2} \cos ^{2} \phi_{6-p}-\left(1-v^{2}\right)^{2}\left(\frac{\partial \phi_{6-p}}{\partial y}\right)^{2}\right]
\end{gathered}
$$


For $\phi_{2-p}$ on the other hand we get,

$$
\begin{aligned}
\left(1-v^{2}\right)^{2} \frac{\partial}{\partial y}\left[r^{\frac{p-3}{2}} \frac{\partial \phi_{2-p}}{\partial y}\right]= & r^{\frac{p-3}{2}} \sin \phi_{2-p} \cos \phi_{2-p}\left[\frac{\omega_{3-p}^{2} d_{3-p}^{2}}{r^{p-3} \sin ^{4} \phi_{2-p}}\right. \\
& -\frac{\omega_{5-p}^{2} d_{5-p}^{2}}{r^{p-3} \cos ^{4} \phi_{2-p} \sin _{4-p}^{2}}-\frac{\omega_{7-p}^{2} d_{7-p}^{2}}{r^{p-3} \cos ^{4} \phi_{2-p} \cos ^{2} \phi_{4-p} \sin ^{2} \phi_{6-p}} \\
& -\frac{\omega_{8-p}^{2} d_{8-p}^{2}}{r^{p-3} \cos ^{4} \phi_{2-p} \cos ^{2} \phi_{4-p} \cos ^{2} \phi_{6-p}}-\omega_{3-p}^{2}+\omega_{5-p}^{2} \sin ^{2} \phi_{4-p} \\
& +\omega_{7-p}^{2} \cos ^{2} \phi_{4-p} \sin ^{2} \phi_{6-p}+\omega_{8-p}^{2} \cos ^{2} \phi_{4-p} \cos ^{2} \phi_{6-p} \\
& \left.-\left(1-v^{2}\right)^{2}\left(\left(\frac{\partial \phi_{4-p}}{\partial y}\right)^{2}+\cos ^{2} \phi_{4-p}\left(\frac{\partial \phi_{6-p}}{\partial y}\right)^{2}\right)\right] .
\end{aligned}
$$

Finally solving for $r$ we get,

$$
\begin{aligned}
& 2\left(1-v^{2}\right)^{2} \frac{\partial}{\partial y}\left[r^{\frac{p-7}{2}} \frac{\partial r}{\partial y}\right]=\left[1-c_{0}^{2} r^{p-7}-\nu_{i}^{2}\left\{1-c_{i}^{2} r^{p-7}\right\}\right] \partial_{r}\left(r^{\frac{7-p}{2}}\right) \\
& \quad+\left(1-v^{2}\right)^{2}\left(\frac{\partial r}{\partial y}\right)^{2} \partial_{r}\left(r^{\frac{p-7}{2}}\right)+\left[\frac{\omega_{3-p}^{2} d_{3-p}^{2}}{r^{p-3} \sin ^{2} \phi_{2-p}}+\frac{\omega_{5-p}^{2} d_{5-p}^{2}}{r^{p-3} \cos ^{2} \phi_{2-p} \sin _{4-p}^{2}}\right. \\
& \quad+\frac{\omega_{7-p}^{2} d_{7-p}^{2}}{r^{p-3} \cos ^{2} \phi_{2-p} \cos ^{2} \phi_{4-p} \sin ^{2} \phi_{6-p}}+\frac{\omega_{8-p}^{2} d_{8-p}^{2}}{r^{p-3} \cos ^{2} \phi_{2-p} \cos ^{2} \phi_{4-p} \cos ^{2} \phi_{6-p}} \\
& \quad-\omega_{3-p}^{2} \sin ^{2} \phi_{2-p}-\omega_{5-p}^{2} \cos ^{2} \phi_{2-p} \sin ^{2} \phi_{4-p}-\omega_{7-p}^{2} \cos ^{2} \phi_{2-p} \cos ^{2} \phi_{4-p} \sin ^{2} \phi_{6-p} \\
& -\omega_{8-p}^{2} \cos ^{2} \phi_{2-p} \cos ^{2} \phi_{4-p} \cos ^{2} \phi_{6-p}+\left(1-v^{2}\right)^{2}\left(\left(\frac{\partial \phi_{2-p}}{\partial y}\right)^{2}+\cos ^{2} \phi_{2-p}\left(\frac{\partial \phi_{4-p}}{\partial y}\right)^{2}\right. \\
& \left.\left.\quad+\cos ^{2} \phi_{2-p} \cos ^{2} \phi_{4-p}\left(\frac{\partial \phi_{6-p}}{\partial y}\right)^{2}\right)\right] \partial_{r}\left(r^{\frac{p-3}{2}}\right) .
\end{aligned}
$$

Clearly these are a set of highly coupled nonlinear equations. It will be hard to solve this system of equations in full generality. Now let's see what we get from Virasoro constraints.

The Virasoro constraint $g_{M N}\left(\partial_{\tau} X^{M} \partial_{\sigma} X^{N}\right)=0$ is given by,

$$
\begin{aligned}
& \left(1-v^{2}\right)^{2}\left[\left(\frac{\partial r}{\partial y}\right)^{2}+r^{2}\left(\frac{\partial \phi_{2-p}}{\partial y}\right)^{2}+r^{2} \cos ^{2} \phi_{2-p}\left(\frac{\partial \phi_{4-p}}{\partial y}\right)^{2}\right. \\
& \left.+r^{2} \cos ^{2} \phi_{2-p} \cos ^{2} \phi_{4-p}\left(\frac{\partial \phi_{6-p}}{\partial y}\right)^{2}\right]=\left(1-\nu_{i}^{2}\right) r^{7-p}+c_{0}^{2}-\nu_{i}^{2} c_{i}^{2}-r^{2} \omega_{3-p}^{2} \sin ^{2} \phi_{2-p} \\
& \quad-r^{2} \omega_{5-p}^{2} \cos ^{2} \phi_{2-p} \sin ^{2} \phi_{4-p}-r^{2} \omega_{7-p}^{2} \cos ^{2} \phi_{2-p} \cos ^{2} \phi_{4-p} \sin ^{2} \phi_{6-p} \\
& \quad-r^{2} \omega_{8-p}^{2} \cos ^{2} \phi_{2-p} \cos ^{2} \phi_{4-p} \cos ^{2} \phi_{6-p}-\frac{\omega_{3-p}^{2} d_{3-p}^{2}}{r^{p-5} \sin ^{2} \phi_{2-p}}-\frac{\omega_{5-p}^{2} d_{5-p}^{2}}{r^{p-5} \cos ^{2} \phi_{2-p} \sin ^{2} \phi_{4-p}} \\
& \quad-\frac{\omega_{8-p}^{2} d_{8-p}^{2}}{r^{p-5} \cos ^{2} \phi_{2-p} \cos ^{2} \phi_{4-p} \sin ^{2} \phi_{6-p}}-\frac{\omega^{p-5} \cos ^{2} \phi_{2-p} \cos ^{2} \phi_{4-p} \cos ^{2} \phi_{6-p}}{\quad} \\
& +\frac{1+v^{2}}{v} r^{\frac{7-p}{2}}\left(-c_{0}+\nu_{i}^{2} c_{i}+\omega_{3-p}^{2} d_{3-p}+\omega_{5-p}^{2} d_{5-p}+\omega_{7-p}^{2} d_{7-p}+\omega_{8-p}^{2} d_{8-p}\right) .
\end{aligned}
$$


Again the Virasoro $g_{M N}\left(\partial_{\tau} X^{M} \partial_{\tau} X^{N}+\partial_{\sigma} X^{M} \partial_{\sigma} X^{N}\right)=0$ becomes,

$$
\begin{aligned}
& \left(1-v^{2}\right)^{2}\left[\left(\frac{\partial r}{\partial y}\right)^{2}+r^{2}\left(\frac{\partial \phi_{2-p}}{\partial y}\right)^{2}+r^{2} \cos ^{2} \phi_{2-p}\left(\frac{\partial \phi_{4-p}}{\partial y}\right)^{2}\right. \\
& \left.+r^{2} \cos ^{2} \phi_{2-p} \cos ^{2} \phi_{4-p}\left(\frac{\partial \phi_{6-p}}{\partial y}\right)^{2}\right]=\left(1-\nu_{i}^{2}\right) r^{7-p}+c_{0}^{2}-\nu_{i}^{2} c_{i}^{2}-r^{2} \omega_{3-p}^{2} \sin ^{2} \phi_{2-p} \\
& \quad-r^{2} \omega_{5-p}^{2} \cos ^{2} \phi_{2-p} \sin ^{2} \phi_{4-p}-r^{2} \omega_{7-p}^{2} \cos ^{2} \phi_{2-p} \cos ^{2} \phi_{4-p} \sin ^{2} \phi_{6-p} \\
& -r^{2} \omega_{8-p}^{2} \cos ^{2} \phi_{2-p} \cos ^{2} \phi_{4-p} \cos ^{2} \phi_{6-p}-\frac{\omega_{3-p}^{2} d_{3-p}^{2}}{r^{p-5} \sin ^{2} \phi_{2-p}}-\frac{\omega_{5-p}^{2} d_{5-p}^{2}}{r^{p-5} \cos ^{2} \phi_{2-p} \sin ^{2} \phi_{4-p}} \\
& -\frac{\omega_{8-p}^{2} d_{8-p}^{2}}{r^{p-5} \cos ^{2} \phi_{2-p} \cos ^{2} \phi_{4-p} \sin ^{2} \phi_{6-p}}-\frac{{ }^{p-5} \cos ^{2} \phi_{2-p} \cos ^{2} \phi_{4-p} \cos ^{2} \phi_{6-p}}{r^{p-p}} \\
& +\frac{4 v}{\left(1+v^{2}\right)} r^{\frac{7-p}{2}}\left(-c_{0}+\nu_{i}^{2} c_{i}+\omega_{3-p}^{2} d_{3-p}+\omega_{5-p}^{2} d_{5-p}+\omega_{7-p}^{2} d_{7-p}+\omega_{8-p}^{2} d_{8-p}\right) .
\end{aligned}
$$

By subtracting the two Virasoro constraints we get the following relation,

$$
-c_{0}+\nu_{i}^{2} c_{i}+\omega_{3-p}^{2} d_{3-p}+\omega_{5-p}^{2} d_{5-p}+\omega_{7-p}^{2} d_{7-p}+\omega_{8-p}^{2} d_{8-p}=0
$$

If we demand that the constraint (2.18) is always satisfied by the solution we are going to present, then we can put this constraint back into the Virasoro, and we obtain,

$$
\begin{aligned}
& \left(1-v^{2}\right)^{2}\left[\left(\frac{\partial r}{\partial y}\right)^{2}+r^{2}\left(\frac{\partial \phi_{2-p}}{\partial y}\right)^{2}+r^{2} \cos ^{2} \phi_{2-p}\left(\frac{\partial \phi_{4-p}}{\partial y}\right)^{2}\right. \\
& \left.+r^{2} \cos ^{2} \phi_{2-p} \cos ^{2} \phi_{4-p}\left(\frac{\partial \phi_{6-p}}{\partial y}\right)^{2}\right]=\left(1-\nu_{i}^{2}\right) r^{7-p}+c_{0}^{2}-\nu_{i}^{2} c_{i}^{2}-r^{2} \omega_{3-p}^{2} \sin ^{2} \phi_{2-p} \\
& \quad-r^{2} \omega_{5-p}^{2} \cos ^{2} \phi_{2-p} \sin ^{2} \phi_{4-p}-r^{2} \omega_{7-p}^{2} \cos ^{2} \phi_{2-p} \cos ^{2} \phi_{4-p} \sin ^{2} \phi_{6-p} \\
& -r^{2} \omega_{8-p}^{2} \cos ^{2} \phi_{2-p} \cos ^{2} \phi_{4-p} \cos ^{2} \phi_{6-p}-\frac{\omega_{3-p}^{2} d_{3-p}^{2}}{r^{p-5} \sin ^{2} \phi_{2-p}}-\frac{\omega_{5-p}^{2} d_{5-p}^{2}}{r^{p-5} \cos ^{2} \phi_{2-p} \sin ^{2} \phi_{4-p}} \\
& \quad-\frac{\omega_{7-p}^{2} d_{7-p}^{2}}{r^{p-5} \cos ^{2} \phi_{2-p} \cos ^{2} \phi_{4-p} \sin ^{2} \phi_{6-p}}-\frac{\omega_{8-p}^{2} d_{8-p}^{2}}{r^{p-5} \cos ^{2} \phi_{2-p} \cos ^{2} \phi_{4-p} \cos ^{2} \phi_{6-p}} \cdot
\end{aligned}
$$

The above Virasoro constraint suggests that we could have some solutions, if we keep only one variable among $r, \phi_{2-p}, \phi_{4-p}, \phi_{6-p}$ and rest of them to be constants. But, putting some of the variables constants will impose non trivial constraints on the system as said earlier. We will analyse all those conditions one by one. 
The conserved charges are given by,

$$
\begin{aligned}
E & =-\int \frac{\partial \mathcal{L}}{\partial \dot{t}} d \sigma=\frac{\sqrt{k}}{2 \pi\left(1-v^{2}\right)} \int\left[r^{\frac{7-p}{2}}-v c_{0}\right] d \sigma \\
P_{i}=\int \frac{\partial \mathcal{L}}{\partial \dot{x}_{i}} d \sigma & =\frac{\sqrt{k} \nu_{i}}{2 \pi\left(1-v^{2}\right)} \int\left[r^{\frac{7-p}{2}}-v c_{i}\right] d \sigma \\
J_{\phi_{3-p}}=\int \frac{\partial \mathcal{L}}{\partial \dot{\phi}_{3-p}} d \sigma & =\frac{\sqrt{k} \omega_{3-p}}{2 \pi\left(1-v^{2}\right)} \int\left[r^{\frac{p-3}{2}} \sin ^{2} \phi_{2-p}-v d_{3-p}\right] d \sigma \\
J_{\phi_{5-p}}=\int \frac{\partial \mathcal{L}}{\partial \dot{\phi}_{5-p}} d \sigma & =\frac{\sqrt{k} \omega_{5-p}}{2 \pi\left(1-v^{2}\right)} \int\left[r^{\frac{p-3}{2}} \cos ^{2} \phi_{2-p} \sin ^{2} \phi_{4-p}-v d_{5-p}\right] d \sigma, \\
J_{\phi_{7-p}}=\int \frac{\partial \mathcal{L}}{\partial \dot{\phi}_{7-p}} d \sigma & =\frac{\sqrt{k} \omega_{7-p}}{2 \pi\left(1-v^{2}\right)} \int\left[r^{\frac{p-3}{2}} \cos ^{2} \phi_{2-p} \cos ^{2} \phi_{4-p} \sin ^{2} \phi_{6-p}-v d_{7-p}\right] d \sigma, \\
J_{\phi_{8-p}}=\int \frac{\partial \mathcal{L}}{\partial \dot{\phi}_{8-p}} d \sigma & =\frac{\sqrt{k} \omega_{8-p}}{2 \pi\left(1-v^{2}\right)} \int\left[r^{\frac{p-3}{2}} \cos ^{2} \phi_{2-p} \cos ^{2} \phi_{4-p} \cos ^{2} \phi_{6-p}-v d_{8-p}\right] d \sigma .
\end{aligned}
$$

Also the deficit angles are given by,

$$
\begin{aligned}
\Delta \phi_{3-p} & =\omega_{3-p} \int \frac{\partial g_{3-p}}{\partial y} d \sigma=\frac{\omega_{3-p}}{1-v^{2}} \int\left[\frac{d_{3-p}}{r^{\frac{p-3}{2}} \sin ^{2} \phi_{2-p}}-v\right] d \sigma \\
\Delta \phi_{5-p} & =\omega_{5-p} \int \frac{\partial g_{5-p}}{\partial y} d \sigma=\frac{\omega_{5-p}}{1-v^{2}} \int\left[\frac{d_{5-p}}{r^{\frac{p-3}{2}} \cos ^{2} \phi_{2-p} \sin ^{2} \phi_{4-p}}-v\right] d \sigma \\
\Delta \phi_{7-p}=\omega_{7-p} \int \frac{\partial g_{7-p}}{\partial y} d \sigma & =\frac{\omega_{7-p}}{1-v^{2}} \int\left[\frac{d_{7-p}}{r^{\frac{p-3}{2}} \cos ^{2} \phi_{2-p} \cos ^{2} \phi_{4-p} \sin ^{2} \phi_{6-p}}-v\right] d \sigma \\
\Delta \phi_{8-p} & =\omega_{8-p} \int \frac{\partial g_{8-p}}{\partial y} d \sigma=\frac{\omega_{8-p}}{1-v^{2}} \int\left[\frac{d_{8-p}}{r^{\frac{p-3}{2}} \cos ^{2} \phi_{2-p} \cos ^{2} \phi_{4-p} \cos ^{2} \phi_{6-p}}-v\right] d \sigma .
\end{aligned}
$$

These relations can be reduced for the D1, D3 and D5- brane backgrounds. In doing so, we should take the terms with only positive subscript. In the next section, we will solve these equations, corresponding to various rotating and orbiting string ansatz explicitly for various D-brane backgrounds as mentioned earlier.

\section{Rotating and orbiting strings in D3-brane background}

Let's start our analysis from D3-brane, because the string equations in the near horizon geometry of D3-brane are integrable. In addition to the giant magnon and single spike solutions of the string, we will further obtain more rotating and orbiting solutions. For $p=3$ equation (2.12) becomes,

$$
\begin{aligned}
\left(1-v^{2}\right)^{2} \frac{\partial}{\partial y}\left[\cos ^{2} \phi_{1} \frac{\partial \phi_{3}}{\partial y}\right]= & \cos ^{2} \phi_{1} \sin \phi_{3} \cos \phi_{3}\left[\frac{\omega_{4}^{2} d_{4}^{2}}{\cos ^{4} \phi_{1} \sin ^{4} \phi_{3}}\right. \\
& \left.-\frac{\omega_{5}^{2} d_{5}^{2}}{\cos ^{4} \phi_{1} \cos ^{4} \phi_{3}}+\omega_{5}^{2}-\omega_{4}^{2}\right]
\end{aligned}
$$


and equation (2.13) becomes,

$$
\begin{aligned}
\left(1-v^{2}\right)^{2} \frac{\partial}{\partial y}\left[\frac{\partial \phi_{1}}{\partial y}\right]= & \sin \phi_{1} \cos \phi_{1}\left[\frac{\omega_{2}^{2} d_{2}^{2}}{\sin ^{4} \phi_{1}}-\frac{\omega_{4}^{2} d_{4}^{2}}{\cos ^{4} \phi_{1} \sin ^{2} \phi_{3}}\right. \\
& \left.-\frac{\omega_{5}^{2} d_{5}^{2}}{\cos ^{4} \phi_{1} \cos ^{2} \phi_{3}}-\omega_{2}^{2}+\omega_{4}^{2} \sin ^{2} \phi_{3}+\omega_{5}^{2} \cos ^{2} \phi_{3}-\left(1-v^{2}\right)^{2}\left(\frac{\partial \phi_{3}}{\partial y}\right)^{2}\right]
\end{aligned}
$$

Equation (2.15) becomes,

$$
2\left(1-v^{2}\right)^{2} \frac{\partial}{\partial y}\left[\frac{1}{r^{2}} \frac{\partial r}{\partial y}\right]=\left[1-\frac{c_{0}^{2}}{r^{4}}-\nu_{i}^{2}\left\{1-\frac{c_{i}^{2}}{r^{4}}\right\}\right] 2 r+\left(1-v^{2}\right)^{2}\left(\frac{\partial r}{\partial y}\right)^{2} \partial_{r}\left(\frac{1}{r^{2}}\right)
$$

It can be noted from these equations of motion that the differential equations corresponding to the radial variable $(r)$ and the angular variables $\left(\phi_{1}, \phi_{3}\right)$ are completely decoupled. We will see later on that this very fact plays an important role in getting exact solutions. Interestingly this happens only for the D3 brane background only. Finally if constraint (2.18) is satisfied then the Virasoro (2.16) becomes,

$$
\begin{aligned}
& \left(1-v^{2}\right)^{2}\left[\left(\frac{\partial r}{\partial y}\right)^{2}+r^{2}\left(\frac{\partial \phi_{1}}{\partial y}\right)^{2}+r^{2} \cos ^{2} \phi_{1}\left(\frac{\partial \phi_{3}}{\partial y}\right)^{2}\right]=\left(1-\nu_{i}^{2}\right) r^{4} \\
& \quad+c_{0}^{2}-\nu_{i}^{2} c_{i}^{2}-r^{2} \omega_{2}^{2} \sin ^{2} \phi_{1}-r^{2} \omega_{4}^{2} \cos ^{2} \phi_{1} \sin ^{2} \phi_{3}-r^{2} \omega_{5}^{2} \cos ^{2} \phi_{1} \cos ^{2} \phi_{3} \\
& \quad-\frac{r^{2} \omega_{2}^{2} d_{2}^{2}}{\sin ^{2} \phi_{1}}-\frac{r^{2} \omega_{4}^{2} d_{4}^{2}}{\cos ^{2} \phi_{1} \sin ^{2} \phi_{3}}-\frac{r^{2} \omega_{5}^{2} d_{5}^{2}}{\cos ^{2} \phi_{1} \cos ^{2} \phi_{3}} .
\end{aligned}
$$

As said earlier, it can be seen from this Virasoro (3.4) that we would get some solutions if we keep only one variable and put rest as constants. But as $r$ equation is decoupled from $\phi_{1}$ and $\phi_{3}$ equations we can relax the above condition as well and can have solutions if we keep $r$ and any one of $\phi_{1}$ and $\phi_{3}$ as variables. In the following we will discuss different possible solutions.

\subsection{Rotating string solutions: constant $\left(r, \phi_{3}\right)$}

In this case $\phi_{1}$ is the only variable and $r$ and $\phi_{3}$ equations will generate constraints on the system. So, from equation (3.1) we get the constraint,

$$
\omega_{4}^{2} d_{4}^{2} \cos ^{4} \phi_{3}-\omega_{5}^{2} d_{5}^{2} \sin ^{4} \phi_{3}=\left(\omega_{4}^{2}-\omega_{5}^{2}\right) \cos ^{4} \phi_{1} \sin ^{4} \phi_{3} \cos ^{4} \phi_{3} .
$$

This constraint (3.5) will imply $\phi_{1}=$ constant, which is our only variable. To avoid this we have to put $\omega_{4}^{2}=\omega_{5}^{4}$. Under this condition the constraint (3.5) becomes,

$$
d_{4}^{2} \cos ^{4} \phi_{3}=d_{5}^{2} \sin ^{4} \phi_{3} .
$$

Using the above constraint (3.6), we get, from equation (3.2),

$$
\left(1-v^{2}\right)^{2} \frac{\partial^{2} \phi_{1}}{\partial y^{2}}=\sin \phi_{1} \cos \phi_{1}\left[\frac{\omega_{2}^{2} d_{2}^{2}}{\sin ^{4} \phi_{1}}-\frac{\omega_{4}^{2} d_{4}^{2}}{\cos ^{4} \phi_{1} \sin ^{4} \phi_{3}}-\omega_{2}^{2}+\omega_{4}^{2}\right] .
$$


Integrating equation (3.7), we have,

$$
\left(1-v^{2}\right)^{2}\left(\frac{\partial \phi_{1}}{\partial y}\right)^{2}=-\frac{\omega_{2}^{2} d_{2}^{2}}{\sin ^{2} \phi_{1}}-\frac{\omega_{4}^{2} d_{4}^{2}}{\cos ^{2} \phi_{1} \sin ^{4} \phi_{3}}-\left(\omega_{2}^{2}-\omega_{4}^{2}\right) \sin ^{2} \phi_{1}+c_{4} .
$$

Equation (3.3) will give the constraint,

$$
\left(1-\nu_{i}^{2}\right) r^{4}=c_{0}^{2}-\nu_{i}^{2} c_{i}^{2} .
$$

Thus the constraints (3.6) and (3.9) fixes the values of the constants $\phi_{3}$ and $r$ respectively. Finally using all the constraints we get from the Virasoro (3.4),

$$
\begin{aligned}
\left(1-v^{2}\right)^{2}\left(\frac{\partial \phi_{1}}{\partial y}\right)^{2}= & -\frac{\omega_{2}^{2} d_{2}^{2}}{\sin ^{2} \phi_{1}}-\frac{\omega_{4}^{2} d_{4}^{2}}{\cos ^{2} \phi_{1} \sin ^{4} \phi_{3}} \\
& -\omega_{2}^{2} \sin ^{2} \phi_{1}-\omega_{4}^{2} \cos ^{2} \phi_{1}+\frac{2\left(c_{0}^{2}-\nu_{i}^{2} c_{i}^{2}\right)}{r^{2}} .
\end{aligned}
$$

Comparing equation (3.8) and equation (3.10) we get,

$$
c_{4}=\frac{2\left(c_{0}^{2}-\nu_{i}^{2} c_{i}^{2}\right)}{r^{2}}-\omega_{2}^{2} .
$$

Using the limit $\frac{\partial \phi_{1}}{\partial y} \rightarrow 0$ as $\phi_{1} \rightarrow \frac{\pi}{2}$ in equation (3.8) we get, $d_{4}=0$ and $c_{4}=\omega_{2}^{2} d_{2}^{2}+\omega_{2}^{2}-\omega_{4}^{2}$. Using this equation (3.8) becomes,

$$
\frac{\partial \phi_{1}}{\partial y}=\frac{\sqrt{\omega_{2}^{2}-\omega_{4}^{2}}}{1-v^{2}} \cot \phi_{1} \sqrt{\sin ^{2} \phi_{1}-\sin ^{2} \phi_{\min }},
$$

where $\sin \phi_{\min }=\frac{\omega_{2} d_{2}}{\sqrt{\omega_{2}^{2}-\omega_{4}^{2}}}$. Below we will discuss two different cases corresponding to giant magnon and single spike solutions for the string separately,

\subsubsection{Giant magnon case}

For $d_{2}=v$,

$$
\Delta \phi_{2}=2 \arccos \left(\sin \phi_{\min }\right) \Rightarrow \sin \phi_{\min }=\cos \frac{\Delta \phi}{2},
$$

where we have taken $\Delta \phi=\Delta \phi_{2}$. It is easy to see that $E, P_{i}$ and $J_{\phi_{2}}$ are divergent independently, but the combination,

$$
\tilde{E}-J_{\phi_{2}}=\frac{\sqrt{k} \omega_{2}}{\pi \sqrt{\omega_{2}^{2}-\omega_{4}^{2}}} \cos \phi_{\min },
$$

is finite, where $\tilde{E}=\frac{\left(1-v^{2}\right) \omega_{2}}{v\left(c_{i}-c_{0}\right)}\left(E-\frac{P_{i}}{\nu_{i}}\right) . J_{\phi_{4}}$ and $J_{\phi_{5}}$ are finite and given by,

$$
J_{\phi_{4}}=\frac{\sqrt{k} \omega_{4}}{\pi \sqrt{\omega_{2}^{2}-\omega_{4}^{2}}} \sin ^{2} \phi_{3} \cos \phi_{\min }, \quad J_{\phi_{5}}=\frac{\sqrt{k} \omega_{5}}{\pi \sqrt{\omega_{2}^{2}-\omega_{4}^{2}}} \cos ^{2} \phi_{3} \cos \phi_{\min } .
$$

Let us define,

$$
J_{\phi}=\omega\left(\frac{J_{\phi_{4}}}{\omega_{4}}+\frac{J_{\phi_{5}}}{\omega_{5}}\right)=\frac{\sqrt{k} \omega}{\pi \sqrt{\omega_{2}^{2}-\omega_{4}^{2}}} \cos \phi_{\min } .
$$


Combining equation (3.14) and equation (3.16), we get the dyonic like giant magnon dispersion relation,

$$
\tilde{E}-J_{\phi_{2}}=\sqrt{J_{\phi}^{2}+\frac{k\left(\omega_{2}^{2}-\omega^{2}\right)}{\pi^{2}\left(\omega_{2}^{2}-\omega_{4}^{2}\right)} \sin ^{2}\left(\frac{\Delta \phi}{2}\right)} .
$$

\subsubsection{Single spike case}

For $d_{2}=\frac{1}{v}, \Delta \phi_{2}, E$ and $P_{i}$ are divergent, but the combination,

$$
\left(\Delta \phi_{2}\right)_{\mathrm{reg}}=\Delta \phi_{2}-\frac{\left(1-v^{2}\right) 2 \pi \omega_{2} d_{2}}{\sqrt{k} v\left(c_{i}-c_{0}\right)}\left(E-\frac{P_{i}}{\nu_{i}}\right)=-2 \arccos \left(\sin \phi_{\min }\right),
$$

is finite, which implies $\sin \phi_{\text {min }}=\cos \frac{(\Delta \phi)_{\text {reg }}}{2}$, where we rename $\left(\Delta \phi_{2}\right)_{\text {reg }}=(\Delta \phi)_{\text {reg. }}$. All the angular momenta are finite here,

$$
\begin{aligned}
J_{\phi_{2}} & =\frac{\sqrt{k} \omega_{2}}{\pi \sqrt{\omega_{2}^{2}-\omega_{4}^{2}}} \cos \phi_{\min } \\
J_{\phi_{4}} & =-\frac{\sqrt{k} \omega_{4}}{\pi \sqrt{\omega_{2}^{2}-\omega_{4}^{2}}} \sin ^{2} \phi_{3} \cos \phi_{\min } \\
J_{\phi_{5}} & =-\frac{\sqrt{k} \omega_{5}}{\pi \sqrt{\omega_{2}^{2}-\omega_{4}^{2}}} \cos ^{2} \phi_{3} \cos \phi_{\min }
\end{aligned}
$$

Again defining the combination,

$$
J_{\phi}=\omega\left(\frac{J_{\phi_{4}}}{\omega_{4}}+\frac{J_{\phi_{5}}}{\omega_{5}}\right)=-\frac{\sqrt{k} \omega}{\pi \sqrt{\omega_{2}^{2}-\omega_{4}^{2}}} \cos \phi_{\min } .
$$

Combining these relations we can write the dispersion relation,

$$
J_{\phi_{2}}=\sqrt{J_{\phi}^{2}+\frac{k\left(\omega_{2}^{2}-\omega^{2}\right)}{\pi^{2}\left(\omega_{2}^{2}-\omega_{4}^{2}\right)} \sin ^{2}\left(\frac{(\Delta \phi)_{\mathrm{reg}}}{2}\right)},
$$

which is nothing but the well known spike dispersion relation. However, there are further rigidly rotating string solutions on this D3-brane which we discuss below.

\subsection{Rotating string solution: constant $\left(r, \phi_{1}\right)$}

In this case $\phi_{3}$ is the only variable. We have from equation (3.1),

$$
\left(1-v^{2}\right)^{2} \frac{\partial}{\partial y}\left[\frac{\partial \phi_{3}}{\partial y}\right]=\sin \phi_{3} \cos \phi_{3}\left[\frac{\omega_{4}^{2} d_{4}^{2}}{\cos ^{4} \phi_{1} \sin ^{4} \phi_{3}}-\frac{\omega_{5}^{2} d_{5}^{2}}{\cos ^{4} \phi_{1} \cos ^{4} \phi_{3}}+\omega_{5}^{2}-\omega_{4}^{2}\right] .
$$

Integrating equation (3.22) we get,

$$
\left(1-v^{2}\right)^{2}\left(\frac{\partial \phi_{3}}{\partial y}\right)^{2}=-\frac{1}{\cos ^{4} \phi_{1}}\left[\frac{\omega_{4}^{2} d_{4}^{2}}{\sin ^{2} \phi_{3}}+\frac{\omega_{5}^{2} d_{5}^{2}}{\cos ^{2} \phi_{3}}\right]+\left(\omega_{5}^{2}-\omega_{4}^{2}\right) \sin ^{2} \phi_{3}+c_{4} .
$$


Again equation (3.2) becomes,

$$
\begin{aligned}
\left(1-v^{2}\right)^{2}\left(\frac{\partial \phi_{3}}{\partial y}\right)^{2}= & -\frac{1}{\cos ^{4} \phi_{1}}\left[\frac{\omega_{4}^{2} d_{4}^{2}}{\sin ^{2} \phi_{3}}+\frac{\omega_{5}^{2} d_{5}^{2}}{\cos ^{2} \phi_{3}}\right]+\frac{\omega_{2}^{2} d_{2}^{2}}{\sin ^{4} \phi_{1}} \\
& -\omega_{2}^{2}+\omega_{4}^{2} \sin ^{2} \phi_{3}+\omega_{5}^{2} \cos ^{2} \phi_{3}
\end{aligned}
$$

Comparing equation (3.23) and (3.24) we get,

$$
c_{4}=2\left(\omega_{4}^{2}-\omega_{5}^{2}\right) \sin ^{2} \phi_{3}-\omega_{2}^{2}+\omega_{5}^{2}+\frac{\omega_{2}^{2} d_{2}^{2}}{\sin ^{4} \phi_{1}} .
$$

This constraint (3.25) implies $\phi_{3}$ is constant, which is our only variable here. To avoid this we must put $\omega_{4}^{2}=\omega_{5}^{2}$. Using this the constraint (3.25) reduces to,

$$
c_{4}=-\omega_{2}^{2}+\omega_{5}^{2}+\frac{\omega_{2}^{2} d_{2}^{2}}{\sin ^{4} \phi_{1}}
$$

and equation (3.23) becomes,

$$
\left(1-v^{2}\right)^{2}\left(\frac{\partial \phi_{3}}{\partial y}\right)^{2}=-\frac{\omega_{4}^{2}}{\cos ^{4} \phi_{1}}\left[\frac{d_{4}^{2}}{\sin ^{2} \phi_{3}}+\frac{d_{5}^{2}}{\cos ^{2} \phi_{3}}\right]+c_{4} .
$$

Equation (3.3) gives the constraint,

$$
\left(1-\nu_{i}^{2}\right) r^{4}=c_{0}^{2}-\nu_{i}^{2} c_{i}^{2}
$$

and finally using all the constraints in Virasoro (3.4) we get,

$$
\begin{aligned}
\left(1-v^{2}\right)^{2}\left(\frac{\partial \phi_{3}}{\partial y}\right)^{2}= & -\frac{\omega_{4}^{2}}{\cos ^{4} \phi_{1}}\left[\frac{d_{4}^{2}}{\sin ^{2} \phi_{3}}+\frac{d_{5}^{2}}{\cos ^{2} \phi_{3}}\right]-\omega_{4}^{2} \\
& +\frac{1}{\cos ^{2} \phi_{1}}\left[\frac{2\left(c_{0}^{2}-\nu_{i}^{2} c_{i}^{2}\right)}{r^{2}}-\omega_{2}^{2} \sin ^{2} \phi_{1}-\frac{\omega_{2}^{2} d_{2}^{2}}{\sin ^{2} \phi_{1}}\right]
\end{aligned}
$$

Again comparing equation (3.27) with equation (3.29) we get,

$$
c_{4}=\frac{1}{\cos ^{2} \phi_{1}}\left[\frac{2\left(c_{0}^{2}-\nu_{i}^{2} c_{i}^{2}\right)}{r^{2}}-\omega_{2}^{2} \sin ^{2} \phi_{1}-\frac{\omega_{2}^{2} d_{2}^{2}}{\sin ^{2} \phi_{1}}\right]-\omega_{4}^{2} .
$$

Finally comparing equation (3.28) with equation (3.30) we get,

$$
2 \omega_{2}^{2} \sin ^{2} \phi_{1}-\omega_{2}^{2}+2 \omega_{4}^{2} \cos ^{2} \phi_{1}+\frac{\omega_{2}^{2} d_{2}^{2}}{\sin ^{4} \phi_{1}}=2 \sqrt{\left(1-\nu_{i}^{2}\right)\left(c_{0}^{2}-\nu_{i}^{2} c_{i}^{2}\right)} .
$$

Using all these constraints finally we get,

$$
\frac{\partial \phi_{3}}{\partial y}=\frac{\sqrt{c_{4}}}{1-v^{2}} \frac{\sqrt{\left(\sin ^{2} \phi_{3}-\sin ^{2} \phi_{\min }\right)\left(\sin ^{2} \phi_{\max }-\sin ^{2} \phi_{3}\right)}}{\sin \phi_{3} \cos \phi_{3}},
$$


where $\sin ^{2} \phi_{\max }+\sin ^{2} \phi_{\min }=1+\frac{\omega_{4}^{2}\left(d_{4}^{2}-d_{5}^{2}\right)}{c_{4}^{2} \cos ^{4} \phi_{1}}$ and $\sin ^{2} \phi_{\max } \sin ^{2} \phi_{\min }=\frac{\omega_{4}^{2} d_{4}^{2}}{c_{4}^{2} \cos ^{4} \phi_{1}}$. In this case, all the conserved charges are finite when we integrate $\phi_{3}$ from $\phi_{\min }$ to $\phi_{\max }$, the explicit expression for these are given by,

$$
E= \pm \frac{\sqrt{k}\left(r^{2}-v c_{0}\right)}{2 \sqrt{c_{4}}}, \quad P_{i}= \pm \frac{\sqrt{k} \nu_{i}\left(r^{2}-v c_{i}\right)}{2 \sqrt{c_{4}}}
$$

we can combine them as

$$
E-\frac{1}{3} \sum_{i=1,2,3} \frac{P_{i}}{\nu_{i}}= \pm \frac{\sqrt{k} v}{2 \sqrt{c_{4}}}\left[\frac{1}{3} \sum_{i} c_{i}-c_{0}\right]
$$

Also,

$$
\begin{aligned}
J_{\phi_{2}} & = \pm \frac{\sqrt{k} \omega_{2}}{2 \sqrt{c_{4}}}\left[\sin ^{2} \phi_{1}-v d_{2}\right] \\
J_{\phi_{4}} & = \pm \frac{\sqrt{k} \omega_{4}}{4 \sqrt{c_{4}}}\left[\cos ^{2} \phi_{1}\left(\sin ^{2} \phi_{\min }+\sin ^{2} \phi_{\max }\right)-2 v d_{4}\right] \\
J_{\phi_{5}} & = \pm \frac{\sqrt{k} \omega_{5}}{4 \sqrt{c_{4}}}\left[2\left(\cos ^{2} \phi_{1}-v d_{5}\right)-\cos ^{2} \phi_{1}\left(\sin ^{2} \phi_{\min }+\sin ^{2} \phi_{\max }\right)\right] .
\end{aligned}
$$

We can also combine,

$$
\frac{J_{\phi_{2}}}{\omega_{2}}+\frac{J_{\phi_{4}}}{\omega_{4}}+\frac{J_{\phi_{5}}}{\omega_{5}}= \pm \frac{\sqrt{k}}{2 \sqrt{c_{4}}}\left[1-v\left(d_{2}+d_{4}+d_{5}\right)\right] .
$$

Combining equation (3.34) and equation (3.36), we get,

$$
E-\frac{1}{3} \sum_{i=1,2,3} \frac{P_{i}}{\nu_{i}}-\sum_{j=2,4,5} \frac{J_{\phi_{j}}}{\omega_{j}}= \pm \frac{\sqrt{k}}{2 \sqrt{c_{4}}}\left[v\left(-c_{0}+\frac{1}{3} \sum_{i} c_{i}+\sum_{j} d_{j}\right)-1\right]
$$

We can't relate this equation with the deficit angles, so we are not writing their expressions explicitly. This is a new kind of rotating string solution which is of the form $E-J=$ constant, for constant $E$ and $J$. One could think them as dual to some chiral primary operators on the gauge theory side, however we would like to mention that the ansatz that we have proposed here are not the typical closed string ansatz, rather they are for the open strings.

\subsection{Orbiting string solution: constant $\left(\phi_{1}, \phi_{3}\right)$}

In this case $r$ is the only variable. From equation (3.1) we have

$$
\omega_{4}^{2} d_{4}^{2} \cos ^{4} \phi_{3}-\omega_{5}^{2} d_{5}^{2} \sin ^{4} \phi_{3}=\left(\omega_{4}^{2}-\omega_{5}^{2}\right) \cos ^{4} \phi_{1} \sin ^{4} \phi_{3} \cos ^{4} \phi_{3} .
$$

Also from equation (3.2) we have,

$$
\begin{aligned}
& \omega_{2}^{2} d_{2}^{2} \sin ^{2} \phi_{3} \cos ^{2} \phi_{3}-\sin ^{4} \phi_{1}\left(\omega_{4}^{2} d_{4}^{2} \cos ^{2} \phi_{3}+\omega_{5}^{2} d_{5}^{2} \sin ^{2} \phi_{3}\right) \\
& \quad=\left(\omega_{2}^{2}-\omega_{4}^{2} \sin ^{2} \phi_{3}-\omega_{5}^{2} \cos ^{2} \phi_{3}\right) \sin ^{4} \phi_{1} \cos ^{4} \phi_{1} \sin ^{2} \phi_{3} \cos ^{2} \phi_{3} .
\end{aligned}
$$


From Virasoro (3.4) we have,

$$
\left(1-v^{2}\right)^{2}\left(\frac{\partial r}{\partial y}\right)^{2}=\left(1-\nu_{i}^{2}\right) r^{4}-c_{4} r^{2}+\left(c_{0}^{2}-\nu_{i}^{2} c_{i}^{2}\right)
$$

where $c_{4}=\omega_{2}^{2} \sin ^{2} \phi_{1}+\omega_{4}^{2} \cos ^{2} \phi_{1} \sin ^{2} \phi_{3}+\omega_{5}^{2} \cos ^{2} \phi_{1} \cos ^{2} \phi_{3}+\frac{\omega_{2}^{2} d_{2}^{2}}{\sin ^{2} \phi_{1}}+\frac{\omega_{4}^{2} d_{4}^{2}}{\cos ^{2} \phi_{1} \sin ^{2} \phi_{3}}+$ $\frac{\omega_{5}^{2} d_{5}^{2}}{\cos ^{2} \phi_{1} \cos ^{2} \phi_{3}}$. Substituting the value of $\frac{\partial r}{\partial y}$ from (3.40) into equation (3.3) we get,

$$
2\left(1-v^{2}\right)^{2} \frac{\partial^{2} r}{\partial y^{2}}=4\left(1-\nu_{i}^{2}\right) r^{3}-2 c_{4} r .
$$

Integrating equation (3.41) we get,

$$
\left(1-v^{2}\right)^{2}\left(\frac{\partial r}{\partial y}\right)^{2}=\left(1-\nu_{i}^{2}\right) r^{4}-c_{4} r^{2}+c_{5}
$$

Comparing equation (3.40) with equation (3.42) we get,

$$
c_{5}=c_{0}^{2}-\nu_{i}^{2} c_{i}^{2} .
$$

So, finally we have

$$
\frac{\partial r}{\partial y}=\frac{\sqrt{1-\nu_{i}^{2}}}{1-v^{2}} \sqrt{\left(r^{2}-a^{2}\right)\left(r^{2}-b^{2}\right)},
$$

where $a^{2}+b^{2}=\frac{c_{4}}{1-\nu_{i}^{2}}$ and $a^{2} b^{2}=\frac{c_{5}}{1-\nu_{i}^{2}}$. Now the conserved charges in this case are given by,

$$
\begin{aligned}
E & =\frac{\sqrt{k}}{\pi \sqrt{1-\nu_{i}^{2}}}\left[I_{1}-v c_{0} I_{2}\right], \\
P_{i} & =\frac{\sqrt{k} \nu_{i}}{\pi \sqrt{1-\nu_{i}^{2}}}\left[I_{1}-v c_{i} I_{2}\right], \\
J_{\phi_{2}} & =\frac{\sqrt{k} \omega_{2}}{\pi \sqrt{1-\nu_{i}^{2}}}\left(\sin ^{2} \phi_{1}-v d_{2}\right) I_{2}, \\
J_{\phi_{4}} & =\frac{\sqrt{k} \omega_{4}}{\pi \sqrt{1-\nu_{i}^{2}}}\left(\cos ^{2} \phi_{1} \sin ^{2} \phi_{3}-v d_{4}\right) I_{2}, \\
J_{\phi_{5}} & =\frac{\sqrt{k} \omega_{5}}{\pi \sqrt{1-\nu_{i}^{2}}}\left(\cos ^{2} \phi_{1} \cos ^{2} \phi_{3}-v d_{5}\right) I_{2},
\end{aligned}
$$

where

$$
\begin{aligned}
I_{1}=\int \frac{r^{2} d r}{\sqrt{\left(r^{2}-a^{2}\right)\left(r^{2}-b^{2}\right)}= \pm b}\left[E\left[i \sinh ^{-1}\left(\frac{i r}{a}\right), \frac{a^{2}}{b^{2}}\right]\right. \\
\left.-F\left[i \sinh ^{-1}\left(\frac{i r}{a}\right), \frac{a^{2}}{b^{2}}\right]\right], \\
I_{2}=\int \frac{d r}{\sqrt{\left(r^{2}-a^{2}\right)\left(r^{2}-b^{2}\right)}}= \pm \frac{1}{b} F\left[\sin ^{-1}\left(\frac{r}{a}\right), \frac{a^{2}}{b^{2}}\right],
\end{aligned}
$$


where $F(\varphi, m)$ and $E(\varphi, m)$ are the incomplete elliptic integrals of first and second kind respectively. Note that in this case we have not use any integration limit. Now we can combine different charges to find a relationship among themselves, firstly combining $E$ and $P_{i}$ we get,

$$
E-\frac{P_{i}}{\nu_{i}}=\frac{\sqrt{k}}{\pi \sqrt{1-\nu_{i}^{2}}} v\left(c_{i}-c_{0}\right) I_{2},
$$

then combining the $J_{\phi}$ 's we get,

$$
\sum_{j=2,4,5} \frac{J_{\phi_{j}}}{\omega_{j}}=\frac{J_{\phi_{2}}}{\omega_{2}}+\frac{J_{\phi_{4}}}{\omega_{4}}+\frac{J_{\phi_{5}}}{\omega_{5}}=\frac{\sqrt{k}}{\pi \sqrt{1-\nu_{i}^{2}}}\left[1-v \sum_{j} d_{j}\right] I_{2},
$$

and combining these two relations we get,

$$
\sum_{j=2,4,5} \frac{J_{\phi_{j}}}{\omega_{j}}=\frac{1-v \sum_{j} d_{j}}{v\left(c_{i}-c_{0}\right)}\left(E-\frac{P_{i}}{\nu_{i}}\right) .
$$

These are some new solutions which are obtained from a particular embedding of worldsheet variables of the string in the stack of D3-brane background. To have a better understanding of these solutions we can use a particular type of integration limit. For example, if we use the limit $\frac{\partial r}{\partial y} \rightarrow 0$ as $r \rightarrow 0$, then from equation (3.42) we immediately get $c_{5}=0$ and it becomes,

$$
\frac{\partial r}{\partial y}=\frac{\sqrt{1-\nu_{i}^{2}}}{1-v^{2}} r \sqrt{r^{2}-r_{0}^{2}}
$$

where $r_{0}=\frac{c_{4}}{1-\nu_{i}^{2}}$. In this case we can integrate $r$ from 0 to $r_{0}$ and find from (3.46) that $I_{1}$ is finite, while $I_{2}$ diverges. This implies all the conserved charges diverge including the deficit angles. However, the relation (3.49) is still true and the solution have the form $E-J=0$ for diverging $E$ and $J$. It will be nice to be able to tell about the corresponding operators in detail.

\section{Strings in D5-brane background}

In this section we wish to study the rotating and orbiting strings in the D5-brane background. For $p=5$ equation (2.12) becomes,

$$
\left(1-v^{2}\right)^{2} \frac{\partial}{\partial y}\left[r \frac{\partial \phi_{1}}{\partial y}\right]=r \sin \phi_{1} \cos \phi_{1}\left[\frac{\omega_{2}^{2} d_{2}^{2}}{r^{2} \sin ^{4} \phi_{1}}-\frac{\omega_{3}^{2} d_{3}^{2}}{r^{2} \cos ^{4} \phi_{1}}+\omega_{3}^{2}-\omega_{2}^{2}\right]
$$

and equation (2.15) becomes,

$$
\begin{aligned}
2\left(1-v^{2}\right)^{2} \frac{\partial}{\partial y}\left[\frac{1}{r} \frac{\partial r}{\partial y}\right]= & \left(1-\nu_{i}^{2}\right)-\frac{c_{0}^{2}-\nu_{i}^{2} c_{i}^{2}}{r^{2}}+\left(1-v^{2}\right)^{2}\left(\frac{\partial r}{\partial y}\right)^{2} \partial_{r}\left(\frac{1}{r}\right) \\
& +\frac{\omega_{2}^{2} d_{2}^{2}}{r^{2} \sin ^{2} \phi_{1}}+\frac{\omega_{3}^{2} d_{3}^{2}}{r^{2} \cos ^{2} \phi_{1}}-\omega_{2}^{2} \sin ^{2} \phi_{1}-\omega_{3}^{2} \cos ^{2} \phi_{1}+(1-v)^{2}\left(\frac{\partial \phi_{1}}{\partial y}\right)^{2}
\end{aligned}
$$


As one can note that these system of equations are coupled and one can not solve for $r$ and $\phi_{1}$ independently as in the case of D3 brane background. Finally if constraint (2.18) is satisfied then the Virasoro (2.16) becomes,

$$
\begin{aligned}
\left(1-v^{2}\right)^{2}\left[\left(\frac{\partial r}{\partial y}\right)^{2}+r^{2}\left(\frac{\partial \phi_{1}}{\partial y}\right)^{2}\right]= & \left(1-\nu_{i}^{2}\right) r^{2}+c_{0}^{2}-\nu_{i}^{2} c_{i}^{2} \\
& -r^{2} \omega_{2}^{2} \sin ^{2} \phi_{1}-r^{2} \omega_{3}^{2} \cos ^{2} \phi_{1}-\frac{\omega_{2}^{2} d_{2}^{2}}{\sin ^{2} \phi_{1}}-\frac{\omega_{3}^{2} d_{3}^{2}}{\cos ^{2} \phi_{1}} .
\end{aligned}
$$

Now by looking at the Virasoro (4.3), to get some solution we have to put one of the variable to be constant. In the following we will discuss the different possible solutions.

\subsection{Rotating string solution: constant $(r)$}

Here $\phi_{1}$ is the only variable, and equation (4.1) becomes,

$$
\left(1-v^{2}\right)^{2} \frac{\partial}{\partial y}\left[\frac{\partial \phi_{1}}{\partial y}\right]=\sin \phi_{1} \cos \phi_{1}\left[\frac{\omega_{2}^{2} d_{2}^{2}}{r^{2} \sin ^{4} \phi_{1}}-\frac{\omega_{3}^{2} d_{3}^{2}}{r^{2} \cos ^{4} \phi_{1}}+\omega_{3}^{2}-\omega_{2}^{2}\right]
$$

Integrating (4.4) we get,

$$
\left(1-v^{2}\right)^{2}\left(\frac{\partial \phi_{1}}{\partial y}\right)^{2}=-\frac{1}{r^{2}}\left[\frac{\omega_{2}^{2} d_{2}^{2}}{\sin ^{2} \phi_{1}}+\frac{\omega_{3}^{2} d_{3}^{2}}{\cos ^{2} \phi_{1}}\right]+\left(\omega_{3}^{2}-\omega_{2}^{2}\right) \sin ^{2} \phi_{1}+c_{6} .
$$

Now from equation (4.2) we have,

$$
\begin{aligned}
\left(1-v^{2}\right)^{2}\left(\frac{\partial \phi_{1}}{\partial y}\right)^{2}= & -\frac{1}{r^{2}}\left[\frac{\omega_{2}^{2} d_{2}^{2}}{\sin ^{2} \phi_{1}}+\frac{\omega_{3}^{2} d_{3}^{2}}{\cos ^{2} \phi_{1}}\right]+\omega_{2}^{2} \sin ^{2} \phi_{1}+\omega_{3}^{2} \cos ^{2} \phi_{1} \\
& -\left(1-\nu_{i}^{2}\right)+\frac{c_{0}^{2}-\nu_{i}^{2} c_{i}^{2}}{r^{2}}
\end{aligned}
$$

Comparing equation (4.5) with equation (4.6) we get,

$$
c_{6}=2\left(\omega_{2}^{2}-\omega_{3}^{2}\right) \sin ^{2} \phi_{1}+\omega_{3}^{2}-\left(1-\nu_{i}^{2}\right)+\frac{c_{0}^{2}-\nu_{i}^{2} c_{i}^{2}}{r^{2}}
$$

This constraint (4.7) implies $\phi_{1}$ is constant which is our only variable. To aviod this we should put $\omega_{2}^{2}=\omega_{3}^{2}$. Under this condition the constraint (4.7) reduces to,

$$
c_{6}=\omega_{2}^{2}-\left(1-\nu_{i}^{2}\right)+\frac{c_{0}^{2}-\nu_{i}^{2} c_{i}^{2}}{r^{2}}
$$

and equation (4.5) reduces to,

$$
\left(1-v^{2}\right)^{2}\left(\frac{\partial \phi_{1}}{\partial y}\right)^{2}=-\frac{\omega_{2}^{2}}{r^{2}}\left[\frac{d_{2}^{2}}{\sin ^{2} \phi_{1}}+\frac{d_{3}^{2}}{\cos ^{2} \phi_{1}}\right]+c_{6}
$$

Finally from Virasoro we have,

$$
\left(1-v^{2}\right)^{2}\left(\frac{\partial \phi_{1}}{\partial y}\right)^{2}=-\frac{\omega_{2}^{2}}{r^{2}}\left[\frac{d_{2}^{2}}{\sin ^{2} \phi_{1}}+\frac{d_{3}^{2}}{\cos ^{2} \phi_{1}}\right]-\omega_{2}^{2}+\left(1-\nu_{i}^{2}\right)+\frac{c_{0}^{2}-\nu_{i}^{2} c_{i}^{2}}{r^{2}}
$$


Again comparing equation (4.9) with equation (4.10) we get,

$$
c_{6}=\left(1-\nu_{i}^{2}\right)+\frac{c_{0}^{2}-\nu_{i}^{2} c_{i}^{2}}{r^{2}}-\omega_{2}^{2} .
$$

Comparing these two $c_{6}$ 's we get the constraint,

$$
\omega_{2}^{2}=\left(1-\nu_{i}^{2}\right), \quad c_{6}=\frac{c_{0}^{2}-\nu_{i}^{2} c_{i}^{2}}{r^{2}} .
$$

Using all these conditions and constraints we get,

$$
\frac{\partial \phi_{1}}{\partial y}=\frac{\sqrt{c_{6}}}{1-v^{2}} \frac{\sqrt{\left(\sin ^{2} \phi_{1}-\sin ^{2} \phi_{\min }\right)\left(\sin ^{2} \phi_{\max }-\sin ^{2} \phi_{1}\right)}}{\sin \phi_{1} \cos \phi_{1}},
$$

where $\sin ^{2} \phi_{\min }+\sin ^{2} \phi_{\max }=1+\frac{\omega_{2}^{2}\left(d_{2}^{2}-d_{3}^{2}\right)}{c_{6} r^{2}}$ and $\sin ^{2} \phi_{\min } \sin ^{2} \phi_{\max }=\frac{\omega_{2}^{2} d_{2}^{2}}{c_{6} r^{2}}$. In this case, all the conserved charges are finite when we integrate $\phi_{1}$ from $\phi_{\min }$ to $\phi_{\max }$, the explicit expression for these are given by,

$$
E= \pm \frac{\sqrt{k}\left(r^{2}-v c_{0}\right)}{2 \sqrt{c_{6}}}, \quad P_{i}= \pm \frac{\sqrt{k} \nu_{i}\left(r^{2}-v c_{i}\right)}{2 \sqrt{c_{6}}}
$$

we can combine them as

$$
E-\frac{1}{5} \sum_{i=1}^{5} \frac{P_{i}}{\nu_{i}}= \pm \frac{\sqrt{k} v}{2 \sqrt{c_{6}}}\left[\frac{1}{5} \sum_{i} c_{i}-c_{0}\right]
$$

Also,

$$
\begin{aligned}
J_{\phi_{2}} & = \pm \frac{\sqrt{k} \omega_{2}}{4 \sqrt{c_{6}}}\left[r\left(\sin ^{2} \phi_{\min }+\sin ^{2} \phi_{\max }\right)-2 v d_{2}\right], \\
J_{\phi_{3}} & = \pm \frac{\sqrt{k} \omega_{3}}{4 \sqrt{c_{6}}}\left[2\left(r-v d_{3}\right)-r\left(\sin ^{2} \phi_{\min }+\sin ^{2} \phi_{\max }\right)\right] .
\end{aligned}
$$

We can also combine,

$$
\frac{J_{\phi_{2}}}{\omega_{2}}+\frac{J_{\phi_{3}}}{\omega_{3}}= \pm \frac{\sqrt{k}}{2 \sqrt{c_{6}}}\left[r-v\left(d_{2}+d_{3}\right)\right]
$$

Combining equation (4.15) and equation (4.17), we get,

$$
E-\frac{1}{5} \sum_{i=1}^{5} \frac{P_{i}}{\nu_{i}}-\sum_{j=2,3} \frac{J_{\phi_{j}}}{\omega_{j}}= \pm \frac{\sqrt{k}}{2 \sqrt{c_{6}}}\left[v\left(-c_{0}+\frac{1}{5} \sum_{i} c_{i}+\sum_{j} d_{j}\right)-r\right] .
$$

As said in the previous section, we can't relate this equation with the deficit angles, so we are not writing their expressions explicitly. Since $r$ is constant in this case, the right hand side of the final equation (4.18) is again constant and this relation is of the type $E-J$ is constant, for $E$ constant and $J$ constant, as we obtain for the case of D3 branes equation (3.37). 


\subsection{Orbiting string solution: constant $\left(\phi_{1}\right)$}

In this case $r$ is the only variable. So, from equation (4.1) we have,

$$
\omega_{2}^{2} d_{2}^{2} \cos ^{4} \phi_{1}-\omega_{3}^{2} d_{3}^{2} \sin ^{4} \phi_{1}=\left(\omega_{2}^{2}-\omega_{3}^{2}\right) r^{2} \sin ^{4} \phi_{1} .
$$

This constraint (4.19) implies $r$ is constant which is our only variable. To avoid this we must put $\omega_{2}^{2}=\omega_{3}^{2}$. Under this condition the constraint (4.19) reduces to,

$$
d_{2}^{2} \cos ^{4} \phi_{1}=d_{3}^{2} \sin ^{4} \phi_{1} .
$$

Now from equation (4.2) we have,

$$
\begin{aligned}
2\left(1-v^{2}\right)^{2} \frac{1}{r} \frac{\partial^{2} r}{\partial y^{2}}= & \left(1-v^{2}\right)^{2} \frac{1}{r^{2}}\left(\frac{\partial r}{\partial y}\right)^{2}+\left(1-\nu_{i}^{2}\right) \\
& -\frac{c_{0}^{2}-\nu_{i}^{2} c_{i}^{2}}{r^{2}}+\frac{\omega_{2}^{2} d_{2}^{2}}{r^{2} \sin ^{4} \phi_{1}}-\omega_{2}^{2} .
\end{aligned}
$$

Again from Virasoro (4.3) we have,

$$
\left(1-v^{2}\right)^{2}\left(\frac{\partial r}{\partial y}\right)^{2}=\left(1-\nu_{i}^{2}\right) r^{2}+\left(c_{0}^{2}-\nu_{i}^{2} c_{i}^{2}\right)-r^{2} \omega_{2}^{2}-\frac{\omega_{2}^{2} d_{2}^{2}}{\sin ^{4} \phi_{1}} .
$$

Substituting the value of $\left(\frac{\partial r}{\partial y}\right)^{2}$ from Virasoro (4.22) into equation (4.21) we get,

$$
\left(1-v^{2}\right)^{2} \frac{\partial^{2} r}{\partial y^{2}}=\left(1-\nu_{i}^{2}-\omega_{2}^{2}\right) r
$$

Integrating (4.23) we get,

$$
\left(1-v^{2}\right)^{2}\left(\frac{\partial r}{\partial y}\right)^{2}=\left(1-\nu_{i}^{2}-\omega_{2}^{2}\right) r^{2}+c_{6}
$$

Comparing equation (4.22) with equation (4.24) we get,

$$
c_{6}=c_{0}^{2}-\nu_{i}^{2} c_{i}^{2}-\frac{\omega_{2}^{2} d_{2}^{2}}{\sin ^{4} \phi_{1}} .
$$

Therefore we have,

$$
\frac{\partial r}{\partial y}=\frac{\sqrt{1-\nu_{i}^{2}-\omega_{2}^{2}}}{1-v^{2}} \sqrt{r^{2}+r_{0}^{2}},
$$

where $r_{0}^{2}=\frac{c_{6}}{1-\nu_{i}^{2}-\omega_{2}^{2}}$. In this case the conserved quantities becomes,

$$
\begin{aligned}
E & =\frac{\sqrt{k}}{\pi \sqrt{1-\nu_{i}^{2}-\omega_{2}^{2}}}\left[I_{1}-v c_{0} I_{2}\right], \\
P_{i} & =\frac{\sqrt{k} \nu_{i}}{\pi \sqrt{1-\nu_{i}^{2}-\omega_{2}^{2}}}\left[I_{1}-v c_{i} I_{2}\right], \\
J_{\phi_{2}} & =\frac{\sqrt{k} \omega_{2}}{\pi \sqrt{1-\nu_{i}^{2}-\omega_{2}^{2}}}\left[\sin ^{2} \phi_{1} I_{1}-v d_{2} I_{2}\right], \\
J_{\phi_{3}} & =\frac{\sqrt{k} \omega_{3}}{\pi \sqrt{1-\nu_{i}^{2}-\omega_{2}^{2}}}\left[\cos ^{2} \phi_{1} I_{1}-v d_{3} I_{2}\right],
\end{aligned}
$$


where

$$
\begin{aligned}
& I_{1}=\int \frac{r d r}{\sqrt{r^{2}+r_{0}^{2}}}=\sqrt{r^{2}+r_{0}^{2}} \\
& I_{2}=\int \frac{d r}{\sqrt{r^{2}+r_{0}^{2}}}=\log \left(r+\sqrt{r^{2}+r_{0}^{2}}\right) .
\end{aligned}
$$

Now we can combine different charges to find a relationship among themselves, firstly combining $E$ and $P_{i}$ we get,

$$
E-\frac{P_{i}}{\nu_{i}}=\frac{\sqrt{k}}{\pi \sqrt{1-\nu_{i}^{2}-\omega_{2}^{2}}} v\left(c_{i}-c_{0}\right) I_{2},
$$

then combining the $J_{\phi}$ 's we get,

$$
\frac{J_{\phi_{2}}}{\omega_{2}}+\frac{J_{\phi_{3}}}{\omega_{3}}=\frac{\sqrt{k}}{\pi \sqrt{1-\nu_{i}^{2}-\omega_{2}^{2}}}\left[I_{1}-v\left(d_{2}+d_{3}\right) I_{2}\right],
$$

and combining these two relations we get,

$$
\sum_{j=2,3} \frac{J_{\phi_{j}}}{\omega_{j}}+\frac{\sum_{j} d_{j}}{c_{i}-c_{0}}\left(E-\frac{P_{i}}{\nu_{i}}\right)=\frac{\sqrt{k}}{\pi \sqrt{1-\nu_{i}^{2}}} I_{1} .
$$

Note that $I_{1}$ is constant once we fix the integration limit and it will be finite unless $r \rightarrow \infty$. So, this relation (4.31) differ from the relation of D3-branes (3.49) by a constant factor.

\section{Strings in D1-brane background}

In this section we proceed to the study of the rotating and orbiting string solutions of the F-string in D1-brane background. In the case, we have from equation (2.12),

$$
\begin{gathered}
\left(1-v^{2}\right)^{2} \frac{\partial}{\partial y}\left[\frac{1}{r} \cos ^{2} \phi_{1} \cos ^{2} \phi_{3} \frac{\partial \phi_{5}}{\partial y}\right]=\frac{1}{r} \cos ^{2} \phi_{1} \cos ^{2} \phi_{3} \sin \phi_{5} \cos \phi_{5} \\
\times\left[\frac{\omega_{6}^{2} d_{6}^{2} r^{2}}{\cos ^{4} \phi_{1} \cos ^{4} \phi_{3} \sin ^{4} \phi_{5}}-\frac{\omega_{7}^{2} d_{7}^{2} r^{2}}{\cos ^{4} \phi_{1} \cos ^{4} \phi_{3} \cos ^{4} \phi_{5}}+\omega_{7}^{2}-\omega_{6}^{2}\right],
\end{gathered}
$$

then from equation (2.13),

$$
\begin{aligned}
\left(1-v^{2}\right)^{2} \frac{\partial}{\partial y}\left[\frac{1}{r} \cos ^{2} \phi_{1} \frac{\partial \phi_{3}}{\partial y}\right]= & \frac{1}{r} \cos ^{2} \phi_{1} \sin \phi_{3} \cos \phi_{3}\left[\frac{\omega_{4}^{2} d_{4}^{2} r^{2}}{\cos ^{4} \phi_{1} \sin ^{4} \phi_{3}}\right. \\
& -\frac{\omega_{6}^{2} d_{6}^{2} r^{2}}{\cos ^{4} \phi_{1} \cos ^{4} \phi_{3} \sin ^{2} \phi_{5}}-\frac{\omega_{7}^{2} d_{7}^{2} r^{2}}{\cos ^{4} \phi_{1} \cos ^{4} \phi_{3} \cos ^{2} \phi_{5}}-\omega_{4}^{2} \\
& \left.+\omega_{6}^{2} \sin ^{2} \phi_{5}+\omega_{7}^{2} \cos ^{2} \phi_{5}-\left(1-v^{2}\right)^{2}\left(\frac{\partial \phi_{5}}{\partial y}\right)^{2}\right]
\end{aligned}
$$


then from equation (2.14),

$$
\begin{aligned}
\left(1-v^{2}\right)^{2} \frac{\partial}{\partial y}\left[\frac{1}{r} \frac{\partial \phi_{1}}{\partial y}\right]= & \frac{1}{r} \sin \phi_{1} \cos \phi_{1}\left[\frac{\omega_{2}^{2} d_{2}^{2} r^{2}}{\sin ^{4} \phi_{1}}-\frac{\omega_{4}^{2} d_{4}^{2} r^{2}}{\cos ^{4} \phi_{1} \sin ^{2} \phi_{3}}\right. \\
& -\frac{\omega_{6}^{2} d_{6}^{2} r^{2}}{\cos ^{4} \phi_{1} \cos ^{2} \phi_{3} \sin ^{2} \phi_{5}}-\frac{\omega_{7}^{2} d_{7}^{2} r^{2}}{\cos ^{4} \phi_{1} \cos ^{2} \phi_{3} \cos ^{2} \phi_{5}}-\omega_{2}^{2} \\
& +\omega_{4}^{2} \sin ^{2} \phi_{3}+\omega_{6}^{2} \cos ^{2} \phi_{3} \sin ^{2} \phi_{5}+\omega_{7}^{2} \cos ^{2} \phi_{3} \cos ^{2} \phi_{5} \\
& \left.-\left(1-v^{2}\right)^{2}\left(\left(\frac{\partial \phi_{3}}{\partial y}\right)^{2}+\cos ^{2} \phi_{3}\left(\frac{\partial \phi_{5}}{\partial y}\right)^{2}\right)\right]
\end{aligned}
$$

and finally from equation (2.15) we get,

$$
\begin{aligned}
2\left(1-v^{2}\right)^{2} \frac{\partial}{\partial y}\left[\frac{1}{r^{3}} \frac{\partial r}{\partial y}\right]= & {\left[1-\frac{c_{0}^{2}}{r^{6}}-\nu_{i}^{2}\left\{1-\frac{c_{i}^{2}}{r^{6}}\right\}\right] \partial_{r}\left(r^{3}\right) } \\
& +\left(1-v^{2}\right)^{2}\left(\frac{\partial r}{\partial y}\right)^{2} \partial_{r}\left(\frac{1}{r^{3}}\right)+\left[\frac{\omega_{2}^{2} d_{2}^{2} r^{2}}{\sin ^{2} \phi_{1}}+\frac{\omega_{4}^{2} d_{4}^{2} r^{2}}{\cos ^{2} \phi_{1} \sin ^{2} \phi_{3}}\right. \\
& +\frac{\omega_{6}^{2} d_{6}^{2} r^{2}}{\cos ^{2} \phi_{1} \cos ^{2} \phi_{3} \sin ^{2} \phi_{5}}+\frac{\omega_{7}^{2} d_{7}^{2} r^{2}}{\cos ^{2} \phi_{1} \cos ^{2} \phi_{3} \cos ^{2} \phi_{5}} \\
& -\omega_{2}^{2} \sin ^{2} \phi_{1}-\omega_{4}^{2} \cos ^{2} \phi_{1} \sin ^{2} \phi_{3}-\omega_{6}^{2} \cos ^{2} \phi_{1} \cos ^{2} \phi_{3} \sin ^{2} \phi_{5} \\
& -\omega_{7}^{2} \cos ^{2} \phi_{1} \cos ^{2} \phi_{3} \cos ^{2} \phi_{5}+\left(1-v^{2}\right)^{2}\left(\left(\frac{\partial \phi_{1}}{\partial y}\right)^{2}+\cos ^{2} \phi_{1}\left(\frac{\partial \phi_{3}}{\partial y}\right)^{2}\right. \\
& \left.\left.+\cos ^{2} \phi_{1} \cos ^{2} \phi_{3}\left(\frac{\partial \phi_{5}}{\partial y}\right)^{2}\right)\right] \partial_{r}\left(\frac{1}{r}\right) .
\end{aligned}
$$

This is a much more complicated system than D5 brane as here all the variables $r, \phi_{1}$, $\phi_{3}$ and $\phi_{5}$ are coupled and we don't expect to solve them independently as this is a non integrable system. In the constraint space,

$$
-c_{0}+\nu_{i}^{2} c_{i}+\omega_{2}^{2} d_{2}+\omega_{4}^{2} d_{4}+\omega_{6}^{2} d_{6}+\omega_{7}^{2} d_{7}=0,
$$

the Virasoro (2.16) becomes,

$$
\begin{aligned}
\left(1-v^{2}\right)^{2}\left[\left(\frac{\partial r}{\partial y}\right)^{2}+r^{2}\left(\frac{\partial \phi_{1}}{\partial y}\right)^{2}+r^{2} \cos ^{2} \phi_{1}\left(\frac{\partial \phi_{3}}{\partial y}\right)^{2}+r^{2} \cos ^{2} \phi_{1} \cos ^{2} \phi_{3}\left(\frac{\partial \phi_{5}}{\partial y}\right)^{2}\right] \\
=\left(1-\nu_{i}^{2}\right) r^{6}+c_{0}^{2}-\nu_{i}^{2} c_{i}^{2}-r^{2} \omega_{2}^{2} \sin ^{2} \phi_{1}-r^{2} \omega_{4}^{2} \cos ^{2} \phi_{1} \sin ^{2} \phi_{3} \\
\quad-r^{2} \omega_{6}^{2} \cos ^{2} \phi_{1} \cos ^{2} \phi_{3} \sin ^{2} \phi_{5}-r^{2} \omega_{7}^{2} \cos ^{2} \phi_{1} \cos ^{2} \phi_{3} \cos ^{2} \phi_{5}-\frac{\omega_{2}^{2} d_{2}^{2} r^{4}}{\sin ^{2} \phi_{1}} \\
\quad-\frac{\omega_{4}^{2} d_{4}^{2} r^{4}}{\cos ^{2} \phi_{1} \sin ^{2} \phi_{3}}-\frac{\omega_{6}^{2} d_{6}^{2} r^{4}}{\cos ^{2} \phi_{1} \cos ^{2} \phi_{3} \sin ^{2} \phi_{5}}-\frac{\omega_{7}^{2} d_{7}^{2} r^{4}}{\cos ^{2} \phi_{1} \cos ^{2} \phi_{3} \cos ^{2} \phi_{5}} .
\end{aligned}
$$

This Virasoro (5.6) suggests that to have some solutions we need to put three of the variables among $r, \phi_{1}, \phi_{3}$ and $\phi_{5}$ to be constants. As three of the variables are being constant here we have to deal with much more constraints. In the following subsection we will discuss one types of such solutions. 


\subsection{Rotating string solution: constant $\left(r, \phi_{3}, \phi_{5}\right)$}

As we put $r, \phi_{3}$ and $\phi_{5}$ as constant, these equations will impose constraints on the system as mentioned previously. In summary, all the constraints are given by,

$$
\begin{array}{rlrl}
\omega_{2}^{2} & =\omega_{4}^{2}=\omega_{6}^{2}=\omega_{7}^{2}, & d_{6}^{2} \cos ^{4} \phi_{5} & =d_{7}^{2} \sin ^{4} \phi_{5}, \\
2 \omega_{2}^{2} & =\left(1-\nu_{i}^{2}\right) r^{4}+\frac{c_{0}^{2}-\nu_{i}^{2} c_{i}^{2}}{r^{2}}, & d_{4}^{2} \cos ^{4} \phi_{3} \sin ^{4} \phi_{5}=d_{6}^{2} \sin ^{4} \phi_{3} .
\end{array}
$$

Under all these constraint (5.7), we have,

$$
\frac{\partial \phi_{1}}{\partial y}=\frac{\omega_{2}}{1-v^{2}} \frac{\sqrt{\left(\sin ^{2} \phi_{1}-\sin ^{2} \phi_{\min }\right)\left(\sin ^{2} \phi_{\max }-\sin ^{2} \phi_{1}\right)}}{\sin \phi_{1} \cos \phi_{1}},
$$

where $\sin ^{2} \phi_{\min }+\sin ^{2} \phi_{\max }=1+r^{2}\left(d_{2}^{2}-\frac{d_{4}^{2}}{\sin ^{4} \phi_{3}}\right)$ and $\sin ^{2} \phi_{\min } \sin ^{2} \phi_{\max }=r^{2} d_{2}^{2}$. In this case the conserved charges are given by,

$$
E= \pm \frac{\sqrt{k}\left(r^{3}-v c_{0}\right)}{2 \omega_{2}}, \quad P_{i}= \pm \frac{\sqrt{k} \nu_{i}\left(r^{3}-v c_{i}\right)}{2 \omega_{2}}
$$

we can combine them as

$$
E-\frac{P_{i}}{\nu_{i}}= \pm \frac{\sqrt{k} v\left(c_{i}-c_{0}\right)}{2 \omega_{2}}
$$

Note that here we didn't take the sum over $\frac{P_{i}}{\nu_{i}}$, because for D1-brane $i=1$. Also,

$$
\begin{aligned}
& J_{\phi_{2}}= \pm \frac{\sqrt{k}}{4}\left[\frac{1}{r}\left(\sin ^{2} \phi_{\min }+\sin ^{2} \phi_{\max }\right)-2 v d_{2}\right] \\
& J_{\phi_{4}}= \pm \frac{\sqrt{k} \omega_{4}}{4 \omega_{2}}\left[2\left(\frac{1}{r} \sin ^{2} \phi_{3}-v d_{4}\right)-\frac{1}{r} \sin ^{2} \phi_{3}\left(\sin ^{2} \phi_{\min }+\sin ^{2} \phi_{\max }\right)\right] \\
& J_{\phi_{6}}= \pm \frac{\sqrt{k} \omega_{6}}{4 \omega_{2}}\left[2\left(\frac{1}{r} \cos ^{2} \phi_{3} \sin ^{2} \phi_{5}-v d_{6}\right)-\frac{1}{r} \cos ^{2} \phi_{3} \sin ^{2} \phi_{5}\left(\sin ^{2} \phi_{\min }+\sin ^{2} \phi_{\max }\right)\right] \\
& J_{\phi_{7}}= \pm \frac{\sqrt{k} \omega_{7}}{4 \omega_{2}}\left[2\left(\frac{1}{r} \cos ^{2} \phi_{3} \cos ^{2} \phi_{5}-v d_{7}\right)-\frac{1}{r} \cos ^{2} \phi_{3} \cos ^{2} \phi_{5}\left(\sin ^{2} \phi_{\min }+\sin ^{2} \phi_{\max }\right)\right] .
\end{aligned}
$$

We can also combine,

$$
\frac{J_{\phi_{2}}}{\omega_{2}}+\frac{J_{\phi_{4}}}{\omega_{4}}+\frac{J_{\phi_{6}}}{\omega_{6}}+\frac{J_{\phi_{7}}}{\omega_{7}}= \pm \frac{\sqrt{k}}{2 \omega_{2}}\left[\frac{1}{r}-v\left(d_{2}+d_{4}+d_{6}+d_{7}\right)\right] .
$$

Combining equation (5.10) and equation (5.12), we get,

$$
E-\frac{P_{i}}{\nu_{i}}-\sum_{j=2,4,6,7} \frac{J_{\phi_{j}}}{\omega_{j}}= \pm \frac{\sqrt{k}}{2 \omega_{2}}\left[v\left(-c_{0}+c_{i}+\sum_{j} d_{j}\right)-\frac{1}{r}\right] .
$$

As $r$ is constant here, the right hand side of (5.13) is constant, and the relation is of similar type that we obtained for D3 and D5 brane background. One will obtain similar kind of solutions in the cases of (i) $\phi_{3}$ as variable and $r, \phi_{1}$ and $\phi_{5}$ as constants and (ii) $\phi_{5}$ as variable and $r, \phi_{1}$ and $\phi_{3}$ as constants. 


\subsection{Orbiting string solution: constant $\left(\phi_{1}, \phi_{3}, \phi_{5}\right)$}

In this section we study the orbiting strings in the D1-brane background. In this case $r$ is the only variable and all angular variable are kept constant. The constraint space is then given by,

$$
\begin{array}{lr}
c_{2}=c_{0}^{2}-\nu_{i}^{2} c_{i}^{2}, \quad \omega_{2}^{2}=\omega_{4}^{2}=\omega_{6}^{2}=\omega_{7}^{2}, & d_{6}^{2} \cos ^{4} \phi_{5}=d_{7}^{2} \sin ^{4} \phi_{5}, \\
d_{4}^{2} \cos ^{4} \phi_{3} \sin ^{4} \phi_{5}=d_{6}^{2} \sin ^{4} \phi_{3}, & d_{2}^{2} \cos ^{4} \phi_{1} \sin ^{4} \phi_{3}=d_{4}^{2} \sin ^{4} \phi_{1} .
\end{array}
$$

So, in this case we have,

$$
\frac{\partial r}{\partial y}=\frac{\sqrt{1-\nu_{i}^{2}}}{1-v^{2}} \sqrt{\left(r^{2}-a^{2}\right)\left(r^{2}-b^{2}\right)\left(r^{2}-c^{2}\right)},
$$

where $a^{2}+b^{2}+c^{2}=\frac{\omega_{2}^{2} d_{2}^{2}}{\left(1-\nu_{i}^{2}\right) \sin ^{4} \phi_{1}}$ and $a^{2} b^{2}+a^{2} c^{2}+b^{2} c^{2}=-\frac{\omega_{2}^{2}}{1-\nu_{i}^{2}}$, and $a^{2} b^{2} c^{2}=-\frac{c_{2}}{1-\nu_{i}^{2}}$. Now the conserved charges in this case are given by,

$$
\begin{aligned}
E & =\frac{\sqrt{k}}{\pi \sqrt{1-\nu_{i}^{2}}}\left[I_{1}-v c_{0} I_{2}\right], \quad P_{i}=\frac{\sqrt{k} \nu_{i}}{\pi \sqrt{1-\nu_{i}^{2}}}\left[I_{1}-v c_{i} I_{2}\right] \\
J_{\phi_{2}} & =\frac{\sqrt{k} \omega_{2}}{\pi \sqrt{1-\nu_{i}^{2}}}\left[\sin ^{2} \phi_{1} I_{3}-v d_{2} I_{2}\right], \quad J_{\phi_{4}}=\frac{\sqrt{k} \omega_{4}}{\pi \sqrt{1-\nu_{i}^{2}}}\left[\cos ^{2} \phi_{1} \sin ^{2} \phi_{3} I_{3}-v d_{4} I_{2}\right] \\
J_{\phi_{6}} & =\frac{\sqrt{k} \omega_{6}}{\pi \sqrt{1-\nu_{i}^{2}}}\left[\cos ^{2} \phi_{1} \cos ^{2} \phi_{3} \sin ^{2} \phi_{5} I_{3}-v d_{6} I_{2}\right] \\
J_{\phi_{7}} & =\frac{\sqrt{k} \omega_{7}}{\pi \sqrt{1-\nu_{i}^{2}}}\left[\cos ^{2} \phi_{1} \cos ^{2} \phi_{3} \cos ^{2} \phi_{5} I_{3}-v d_{7} I_{2}\right]
\end{aligned}
$$

where

$$
\begin{aligned}
I_{1}= & \int \frac{r^{3} d r}{\sqrt{\left(r^{2}-a^{2}\right)\left(r^{2}-b^{2}\right)\left(r^{2}-c^{2}\right)}} \\
= & \frac{\sqrt{\left(r^{2}-b^{2}\right)\left(r^{2}-c^{2}\right)}}{\sqrt{r^{2}-a^{2}}}-\sqrt{b^{2}-a^{2}} E\left[i \sinh ^{-1}\left(\frac{\sqrt{a^{2}-b^{2}}}{\sqrt{r^{2}-a^{2}}}\right), \frac{a^{2}-c^{2}}{a^{2}-b^{2}}\right] \\
& -\frac{b^{2}}{\sqrt{b^{2}-a^{2}}} F\left[i \sinh ^{-1}\left(\frac{\sqrt{a^{2}-b^{2}}}{\sqrt{r^{2}-a^{2}}}\right), \frac{a^{2}-c^{2}}{a^{2}-b^{2}}\right], \\
I_{2}= & \int \frac{d r}{\sqrt{\left(r^{2}-a^{2}\right)\left(r^{2}-b^{2}\right)\left(r^{2}-c^{2}\right)}} \\
= & \frac{1}{b \sqrt{c^{2}-a^{2}}} F\left[\sin ^{-1}\left(\sqrt{\frac{r^{2}\left(c^{2}-a^{2}\right)}{c^{2}\left(r^{2}-a^{2}\right)}}\right), \frac{\left(a^{2}-b^{2}\right) c^{2}}{b^{2}\left(a^{2}-c^{2}\right)}\right], \\
I_{3}= & \int \frac{d r}{\sqrt{r\left(r^{2}-a^{2}\right)\left(r^{2}-b^{2}\right)\left(r^{2}-c^{2}\right)}} \\
= & \frac{1}{c^{2} \sqrt{a^{2}-c^{2}}} \Pi\left[1-\frac{b^{2}}{c^{2}}, \sin ^{-1}\left(\sqrt{\frac{r^{2}-c^{2}}{b^{2}-c^{2}}}\right), \frac{b^{2}-c^{2}}{a^{2}-c^{2}}\right],
\end{aligned}
$$


where $F(\varphi, m), E(\varphi, m)$ and $\Pi(n, \varphi, m)$ are the incomplete elliptic integrals of first, second and third kind respectively. We have to find out which integration limits are useful in this case. However, we can combine different charges to find a relationship among themselves, firstly combining $E$ and $P_{i}$ we get,

$$
E-\frac{P_{i}}{\nu_{i}}=\frac{\sqrt{k}}{\pi \sqrt{1-\nu_{i}^{2}}} v\left(c_{i}-c_{0}\right) I_{2},
$$

then combining the $J_{\phi}$ 's we get,

$$
\frac{J_{\phi_{2}}}{\omega_{2}}+\frac{J_{\phi_{4}}}{\omega_{4}}+\frac{J_{\phi_{6}}}{\omega_{6}}+\frac{J_{\phi_{7}}}{\omega_{7}}=\frac{\sqrt{k}}{\pi \sqrt{1-\nu_{i}^{2}}}\left[I_{3}-v\left(d_{2}+d_{4}+d_{6}+d_{7}\right) I_{2}\right],
$$

and combining these two relations we get,

$$
\sum_{j=2,4,6,7} \frac{J_{\phi_{j}}}{\omega_{j}}+\frac{\sum_{j} d_{j}}{c_{i}-c_{0}}\left(E-\frac{P_{i}}{\nu_{i}}\right)=\frac{\sqrt{k}}{\pi \sqrt{1-\nu_{i}^{2}}} I_{3} .
$$

Unless we find the value of $I_{3}$ using appropriate integration limit, we can not comment on the nature of this relation (5.20) in detail. However, this relation seems to be different from (3.49) and (4.31) of D3 and D5-brane backgrounds.

\section{Conclusion}

This paper is devoted to study various rigidly rotating and orbiting solutions of the Fstring in various Dp-brane backgrounds in type IIB string theory. As the string equations of motion in general Dp-brane backgrounds are non integrable (except for D3 branes), we do not except to find solutions in full generality. We investigate various subspaces of Dp-brane background and found two kinds of solutions, namely the rotating string and the orbiting string solutions. For the D3-brane background we find two classes of rotating string solutions. First one is the well known giant magnon and single spike solution. Second one is a new kind of rotating string solution for which $E-J=$ constant for constant $E$ and $J$. In fact it is the second kind of solution which has similar structure for all the D-brane backgrounds we have considered here. Further we have found another class of solutions which are the so called orbiting string solutions. These are the string configurations where the string orbit around the core of Dp-brane. It would be interesting to study how orbiting strings differ for different Dp-branes by putting appropriate integration limits. Looking for the dual gauge theory operators for these kind of string states will be of interest. Further one would like to study the rotating string solution in some intersecting brane backgrounds with mixed flux and see if one gets the giant magnon and single spike like solutions. It will also be interesting to study the D-string solutions in Dp-brane background.

Open Access. This article is distributed under the terms of the Creative Commons Attribution License (CC-BY 4.0), which permits any use, distribution and reproduction in any medium, provided the original author(s) and source are credited. 


\section{References}

[1] J.M. Maldacena, The large-N limit of superconformal field theories and supergravity, Int. J. Theor. Phys. 38 (1999) 1113 [hep-th/9711200] [INSPIRE].

[2] S.S. Gubser, I.R. Klebanov and A.M. Polyakov, Gauge theory correlators from noncritical string theory, Phys. Lett. B 428 (1998) 105 [hep-th/9802109] [INSPIRE].

[3] E. Witten, Anti-de Sitter space and holography, Adv. Theor. Math. Phys. 2 (1998) 253 [hep-th/9802150] [INSPIRE].

[4] D.E. Berenstein, J.M. Maldacena and H.S. Nastase, Strings in flat space and pp waves from $N=4$ super Yang-Mills, JHEP 04 (2002) 013 [hep-th/0202021] [INSPIRE].

[5] S.S. Gubser, I.R. Klebanov and A.M. Polyakov, A semiclassical limit of the gauge/string correspondence, Nucl. Phys. B 636 (2002) 99 [hep-th/0204051] [INSPIRE].

[6] J.A. Minahan and K. Zarembo, The Bethe ansatz for $N=4$ super Yang-Mills, JHEP 03 (2003) 013 [hep-th/0212208] [INSPIRE].

[7] V.A. Kazakov, A. Marshakov, J.A. Minahan and K. Zarembo, Classical/quantum integrability in AdS/CFT, JHEP 05 (2004) 024 [hep-th/0402207] [INSPIRE].

[8] N. Beisert, V. Dippel and M. Staudacher, A novel long range spin chain and planar $N=4$ super Yang-Mills, JHEP 07 (2004) 075 [hep-th/0405001] [INSPIRE].

[9] G. Arutyunov, S. Frolov and M. Staudacher, Bethe ansatz for quantum strings, JHEP 10 (2004) 016 [hep-th/0406256] [INSPIRE].

[10] N. Beisert et al., Review of AdS/CFT Integrability: An Overview, Lett. Math. Phys. 99 (2012) 3 [arXiv:1012.3982] [InSPIRE].

[11] A. Armoni, J.L.F. Barbon and A.C. Petkou, Orbiting strings in AdS black holes and $N=4$ SYM at finite temperature, JHEP 06 (2002) 058 [hep-th/0205280] [INSPIRE].

[12] S.A. Hartnoll and C. Núñez, Rotating membranes on $G_{2}$ manifolds, logarithmic anomalous dimensions and $N=1$ duality, JHEP 02 (2003) 049 [hep-th/0210218] [INSPIRE].

[13] S. Ryang, Rotating and orbiting strings in the near horizon brane backgrounds, JHEP 04 (2003) 045 [hep-th/0303237] [INSPIRE].

[14] J. Engquist, J.A. Minahan and K. Zarembo, Yang-Mills duals for semiclassical strings on $A d S_{5} \times S^{5}, J H E P 11$ (2003) 063 [hep-th/0310188] [INSPIRE].

[15] M. Smedback, Pulsating strings on $A d S_{5} \times S^{5}$, JHEP 07 (2004) 004 [hep-th/0405102] [INSPIRE].

[16] M. Kruczenski, Spiky strings and single trace operators in gauge theories, JHEP 08 (2005) 014 [hep-th/0410226] [INSPIRE].

[17] N.P. Bobev, H. Dimov and R.C. Rashkov, Semiclassical strings in Lunin-Maldacena background, Bulg. J. Phys. 35 (2008) 274 [hep-th/0506063] [INSPIRE].

[18] D.M. Hofman and J.M. Maldacena, Giant Magnons, J. Phys. A 39 (2006) 13095 [hep-th/0604135] [INSPIRE].

[19] N. Dorey, Magnon Bound States and the AdS/CFT Correspondence, J. Phys. A 39 (2006) 13119 [hep-th/0604175] [INSPIRE].

[20] H.-Y. Chen, N. Dorey and K. Okamura, Dyonic giant magnons, JHEP 09 (2006) 024 [hep-th/0605155] [INSPIRE]. 
[21] N.P. Bobev and R.C. Rashkov, Multispin Giant Magnons, Phys. Rev. D 74 (2006) 046011 [hep-th/0607018] [INSPIRE].

[22] M. Kruczenski, J. Russo and A.A. Tseytlin, Spiky strings and giant magnons on $S^{5}$, JHEP 10 (2006) 002 [hep-th/0607044] [INSPIRE].

[23] H.-Y. Chen, N. Dorey and K. Okamura, On the scattering of magnon boundstates, JHEP 11 (2006) 035 [hep-th/0608047] [INSPIRE].

[24] S. Hirano, Fat magnon, JHEP 04 (2007) 010 [hep-th/0610027] [INSPIRE].

[25] S. Ryang, Three-spin giant magnons in $A d S_{5} \times S^{5}$, JHEP 12 (2006) 043 [hep-th/0610037] [INSPIRE].

[26] R. Ishizeki and M. Kruczenski, Single spike solutions for strings on $S^{2}$ and $S^{3}$, Phys. Rev. D 76 (2007) 126006 [arXiv:0705.2429] [INSPIRE].

[27] N.P. Bobev and R.C. Rashkov, Spiky strings, giant magnons and $\beta$-deformations, Phys. Rev. D 76 (2007) 046008 [arXiv: 0706. 0442] [INSPIRE].

[28] D.M. Hofman and J.M. Maldacena, Reflecting magnons, JHEP 11 (2007) 063 [arXiv:0708.2272] [INSPIRE].

[29] R. Ishizeki, M. Kruczenski, M. Spradlin and A. Volovich, Scattering of single spikes, JHEP 02 (2008) 009 [arXiv: 0710.2300] [INSPIRE].

[30] N. Dorey and K. Okamura, Singularities of the Magnon Boundstate S-matrix, JHEP 03 (2008) 037 [arXiv:0712.4068] [INSPIRE].

[31] J.R. David and B. Sahoo, Giant magnons in the D1-D5 system, JHEP 07 (2008) 033 [arXiv: 0804.3267] [INSPIRE].

[32] B.-H. Lee, K.L. Panigrahi and C. Park, Spiky Strings on $A d S_{4} \times C P^{3}$, JHEP 11 (2008) 066 [arXiv: 0807.2559] [INSPIRE].

[33] S. Biswas and K.L. Panigrahi, Spiky Strings on NS5-branes, Phys. Lett. B 701 (2011) 481 [arXiv: 1103.6153] [INSPIRE].

[34] K.L. Panigrahi, P.M. Pradhan and P.K. Swain, Rotating Strings in $A d S_{4} X C P^{3}$ with $B_{N S}$ holonomy, JHEP 01 (2012) 113 [arXiv:1109.2458] [INSPIRE].

[35] S. Biswas and K.L. Panigrahi, Spiky Strings on I-brane, JHEP 08 (2012) 044 [arXiv: 1206.2539] [INSPIRE].

[36] A. Stepanchuk and A.A. Tseytlin, On (non)integrability of classical strings in p-brane backgrounds, J. Phys. A 46 (2013) 125401 [arXiv:1211.3727] [InSPIRE].

[37] S. Biswas and K.L. Panigrahi, Semiclassical Strings on Curved Branes, JHEP 10 (2013) 106 [arXiv:1307.4926] [INSPIRE].

[38] A. Banerjee, S. Biswas and K.L. Panigrahi, Semiclassical Strings in Supergravity PFT, Eur. Phys. J. C 74 (2014) 3115 [arXiv:1403.7358] [INSPIRE]. 